\title{
Interrelationships of striate and extrastriate cortex with the primary relay sites of the visual pathway
}

\author{
L. J. GAREY, E. G. JONES, AND T. P. S. POWELl \\ From the Department of Human Anatomy, Oxford
}

Several recent studies have indicated that the optic tectum or superior colliculus may serve more important and diverse functions than was formerly considered (Denny-Brown, 1962; Sprague and Meikle, 1965) and there is also evidence that the cortical connexions of the colliculus are significant in such functions (Sprague, 1966a, b). It has been established for some time that the superior colliculus receives a substantial projection from the neocortex (Meikle and Sprague, 1964), but relatively little attention has been paid to the organization that may be present in this system. The finding in the avian brain of a precise relationship between the representation of the retina upon the optic tectum and the origin of one group of tectal efferents (McGill, Powell, and Cowan, 1966), taken together with the well-ordered representation of the retina upon both the superior colliculus (Apter, 1945) and the visual cortex in the cat (Talbot and Marshall, 1941; Bilge, Seneviratne, and Whitteridge, 1963; Hubel and Wiesel, 1965) raises the question whether a comparably close relationship might not be present in the termination of the two distinct groups of afferents to the superior colliculus from the visual pathway-from the retina and from the visual cortex. As the architectonic subdivisions of the visual cortex (Otsuka and Hassler, 1962) have been shown to be functionally different (Hubel and Wiesel, 1965), and because there are separate representations of the retina in these areas, there is also the possibility that each of these areas projects independently upon the colliculus. A further aspect of the projection of the neocortex upon the tectum that should be considered is the relationship of the origin of those cortico-tectal fibres from 'nonvisual' parts of the cortex to such functional areas as the auditory and somatic sensory areas and the motor cortex, and also the relationship of the termination of these fibres to that of the afferent fibres to the superior colliculus from ascending sensory pathways.

The present investigation has been mainly concerned with these problems of the projection of the neocortex upon the superior colliculus in the cat
(Garey, 1965), but as our material has also provided evidence on the cortical and other subcortical connexions of the visual area, certain of these findings have been included here because of their close interrelationship. The experimental results are presented and discussed in three sections, and the possible significance of these and other recent findings in the thalamocortical and mesencephalic connexions of the visual system will be finally considered in relation to the function of the superior colliculus. In the meantime Lund $(1964,1966)$ has published a study of the occipito-tectal pathway in the rat in which he found that "areas of cortex project to areas of colliculus receiving fibres from the same areas of retina', and Kuypers and Lawrence (1967) have described the organization in the projection of the cerebral cortex upon the brain-stem in the monkey.

\section{MATERIAL AND METHODS}

The brains of 36 cats were used for this investigation. The animals were anaesthetized with intraperitoneal Nembutal and operated upon with strict aseptic precautions. Lesions varying in site and extent were made in the cerebral cortex either by subpial suction or by pial devascularization. After it had been shown that the cortical projection upon the superior colliculus is strictly unilateral, lesions were placed in both hemispheres of a certain number of animals, but these were always at distinctly different antero-posterior or medio-lateral levels. After survival periods of between three and seven days the animals were again anaesthetized and perfused with saline and $10 \%$ formalin. The brains were removed and photographed to show the site of damage, and after a further period of fixation were cut at $25 \mu$ on a freezing microtome; the majority were cut in the coronal plane, but four were cut sagittally. A 1 in 10 series of sections was stained with the method of Nauta and Gygax (1954) and over the extent of the lesions and the lateral geniculate nucleus another series was stained by the Nissl method.

\section{THE CORTICO-TECTAL PROJECTION}

RESULTS In the brains with lesions in the visual cortex it was almost always possible, in the Nissl 
sections, to define the boundaries of the three architectonic subdivisions (Otsuka and Hassler, 1962), and the extent of the damage has therefore been described with reference to these. In many of the experiments the lesion either directly damaged more than one area or, through involvement of the underlying white matter, made it difficult to determine the total extent of the interruption of fibres. About one third of the lesions were, however, unquestionably restricted to one or other of these areas.

The first two experiments demonstrate certain features of the course and termination of the fibres passing from the visual cortex to the superior colliculus. In experiment 1 there is a relatively large lesion in the post-lateral gyrus extending for a few $\mathrm{mm}$ in front of and behind the posteromedial angle of the hemisphere (Fig. 1): it involves the dorso-lateral part of area 17 and the medial twothirds of area 18 and, as there is minimal extension into the underlying white matter, it is probable that fibres from the medial part of area 17 and from area 19 have not been interrupted. Degenerated fibres leave the damaged area of cortex in a clearly perpendicular direction and can be traced rostrally to the internal capsule. The majority of these pass medially into the hilum of the lateral geniculate nucleus. On more caudal sections these fibres become more medially situated and then turn vertically downwards between the medial aspect of the main laminae and the medial interlaminar nucleus of the lateral geniculate body and through the posterior nucleus of the thalamus to reach the pretectum and the lateral brachium of the superior colliculus; a few enter the medial brachium. From the brachia and pretectum they enter the ventral part of the stratum opticum and the stratum zonale of the superior colliculus. Degeneration of fibres and terminals is found in the superficial four layers of the colliculus (see Fig. 13): in the stratum zonale there are horizontally disposed fragmented fibres, and in the stratum griseum superficiale there is very dense pericellular degeneration in its ventral half with occasional break-up of radially disposed fibres in its dorsal half; the degeneration in the strata opticum and griseum intermediale becomes progressively less dense as followed deeply. This degeneration is confined to the anterior part of the colliculus where it affects most of its medio-lateral extent, only the medial and lateral edges being free. In experiment 78 the brain was cut sagittally after a large lesion had been made in the lateral and postlateral gyri, involving areas 17 and 18 with minimal extension into the underlying white matter. In these sagittal sections certain features of the course of the cortico-tectal fibres are clearly seen (Fig. 2): where they turn medially, dorsal to the anterior half of the lateral geniculate nucleus, they are cut more or less $Z$ transversely, and are quite distinct from other degenerating fascicles which continue ventrally into the cerebral peduncle; at the medial end of the lateral geniculate nucleus they can be seen turning ventrally and posteriorly through the posterior nucleus to reach the pretectum and brachia of the superior colliculus, from which they continue into the stratum zonale and the ventral part of the stratum opticum in both of which they pass horizontally backwards; numerous fragmented fibres turn off superficially, more or less at right angles, to enter the deep half of the stratum griseum superficiale where there is a dense pericellular plexus, and a relatively clear zone in the superficial third with only a few radially directed fibres crossing it. This pattern of degeneration is found in the anterior half of the superior colliculus, and except for its most medial part the whole medio-lateral extent is affected. In both these brains no degeneration is found in the contra-lateral colliculi nor in any other subcortical structure on the opposite side. In a few experiments sections of the superior colliculus were stained by the Glees method (1946), but vers ? few degenerating fibres and boutons were seen- $\vec{\oplus} \vec{\omega}$ certainly far less than would be expected from th $\Phi^{\circ}$. density of the degeneration shown by the Nauto technique (Beresford, 1963). The fact that degenera $\rightarrow$ tion is found only in the anterior parts of the colliculi after these large, but partial, lesions of the visual cortex, indicates that this projection is topically organized. Before considering the detail $\bar{s}^{+}$. of this organization one experiment will be described to show that areas 17 and 18 of the visual cortex are independently related to the superior colliculus.

In experiment 98, lesions were placed in the visual cortex of both hemispheres; on the right side there is a small area of damage restricted to area 17; it is on the medial surface of the hemisphere, a little anterior to the level of the splenium of the corpus callosum, and it involves the cortex of the walls of the suprasplenial sulcus; there is no extension into the white matter (Fig. 3). The degeneration in the superior colliculus occupies a narrow band about the middle of its lateral half; it is more medial anteriorly, and from here it is directed posterolaterally but does not reach the lateral margin. The intensity of the degeneration in the strata zonale, griseum superficiale, and opticum is about same as after the larger lesions already described, but there is less in the stratum griseum intermediale. In the left hemisphere the lesion occupies a very similar antero-posterior extent to that in the previous brain, but it is in the lateral gyrus on the dorsal surface. It is in the form of a narrow strip which 


\section{KEY TO ABBREVIATIONS}

AI stratum album intermediate of superior colliculus

D dorsal nucleus of lateral geniculate body

I stratum griseum intermediale of superior colliculus

$L \quad$ nucleus lateralis posterior of thalamus

$O \quad$ stratum opticum of superior colliculus

$P \quad$ pulvinar

$P R \quad$ stratum griseum profundum of superior colliculus

$P T$ pretectum

$S \quad$ stratum griseum superficiale of superior colliculus

SS suprasylvian area

$V \quad$ ventral nucleus of lateral geniculate body

$Z \quad$ stratum zonale of superior colliculus

$17)$

$18\}$ Area 17,18 and 19 of visual cortex.

$19\}$
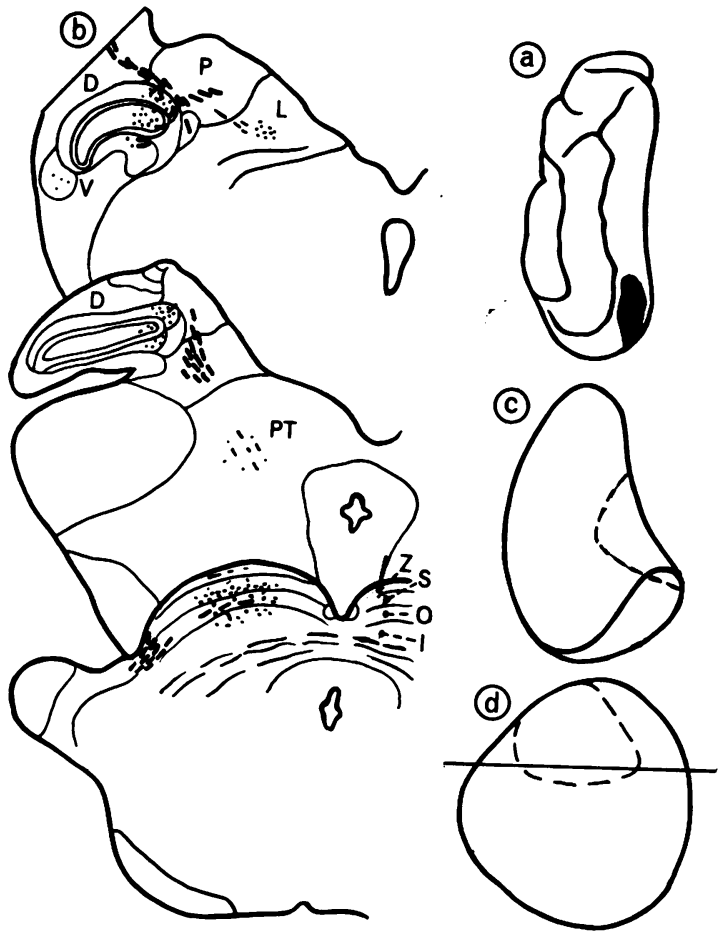

destroys the medial part of area 18 , and there is no involvement of the underlying white matter. In the st., srior colliculus of this side there is an area of degeneration of about the same size and at the same antero-posterior extent as on the right side. For the most part, however, it is more laterally situated, although posteriorly they abut on each other, and there is again little degeneration in the stratum griseum intermediale.

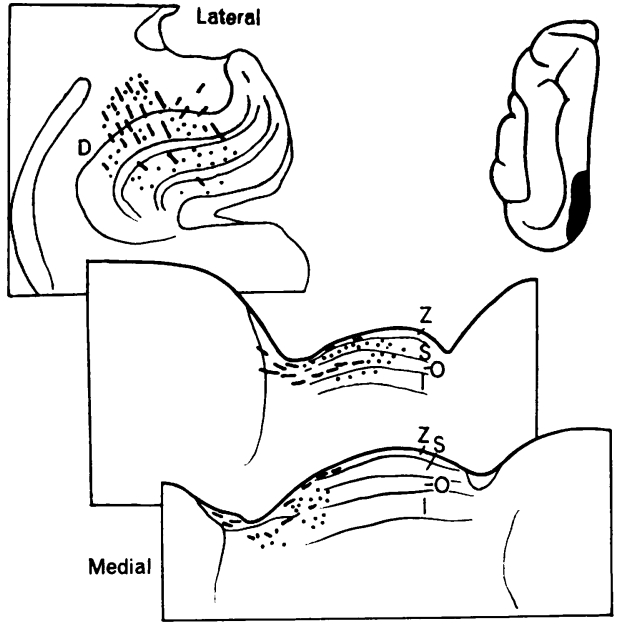

FIG. 2. The lesion in experiment 78 and the ensuing fibre and terminal degeneration on sagittal sections of the caudal thalamus and brain stem.

FIG. 1. To show the lesion in the visual cortex (a) in experiment 1 , and the resulting axonal and terminal degeneration on coronal sections of the caudal thalamus and superior colliculus (b).

The distribution of the degeneration in the lateral geniculate nucleus and in the superior colliculus are shown on orthographic reconstructions of the dorsal surfaces of these structures in (c) and (d) (the horizontal line on the superior colliculus indicates the level of the section).

In this and most of the subsequent figures the lesions have been shown as if made in the left hemisphere, the axonal degeneration is represented by dashes and the terminal degeneration by stippling.

There are 10 further hemispheres in which damage is confined to different parts of the cortex of area 17. The surface extent of these lesions is quite small, and their positions and appearance on sections are shown in Fig. 4; the extent of the damage in the right hemisphere of cat 32 is particularly small, being between 1 and $2 \mathrm{~mm}$ in diameter. The areas of degeneration in the colliculi of these brains are all sharply localized, are easy to identify, 
and are more or less proportional to the extent of the lesions. It is clear from these experiments that the projection of area 17 upon the superficial layers of the superior colliculus is precisely organized. When the anterior part of this area is damaged, as in cats $5 \mathrm{~L}$ and $66 \mathrm{R}$, the degeneration is found close to the lateral margin of the colliculus, but after the posteriorly situated lesion of experiment 33, the medial part is affected. The difference in the projection between medial and lateral parts of area 17 has to be considered at different anteroposterior levels. In the anterior half of this area, damage of its lateral part results in degeneration in anterior and lateral parts of the colliculus; involvement of more medial parts of the cortex always

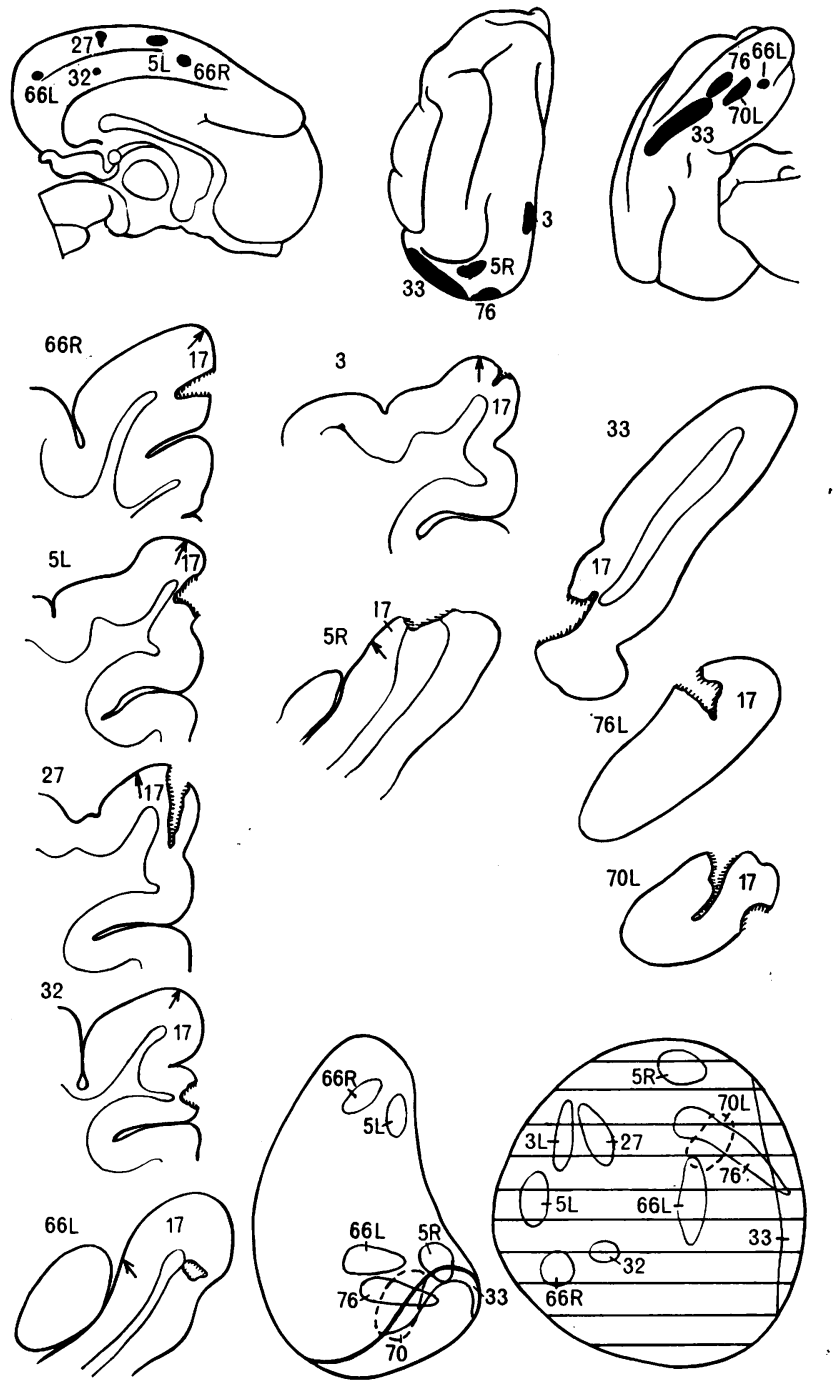

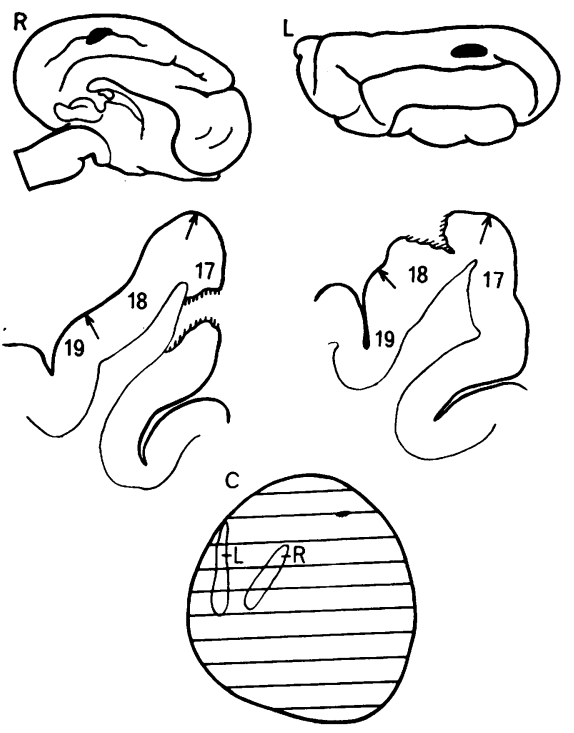

FIG. 3. The lesion in area 17 of the right hemisphere $(R)$ and in area 18 of the left hemisphere $(L)$ of experiment 98 , together wit the distribution of the degeneration in the $\overrightarrow{\mathbb{D}} \vec{\omega}$ superior colliculus $(C)$ due to these two lesion or The horizontal lines on the superior colliculus $\mathrm{Q}$ 을 are at the levels of the sections of a standar series which were used to make the reconstruction of the dorsal surface, and the have been shown in this and subsequent figures. $\overrightarrow{0}$ in order to facilitate comparison of the areas $\bar{x}$ of degeneration.

FIG. 4. The site and extent of lesions restricted to area 17, and the distribution of the degeneration in the lateral geniculate nucleus and superior colliculus in these experiments.

results in the degeneration being more posteriorly and medially situated as compared with that due to a laterally placed lesion at the same antero-posterior level, but it is still in the lateral half of the colliculus. This can be seen by compar- 3 . ing the findings in experiments $5 \mathrm{~L}$ and $66 \mathrm{R}$ at one level and experiments 3 and 27 at 3 another. In the posterior half of area 17, 0 however, the laterally placed lesions of experiments $5 R, 76$, and 33 have produced $\frac{7}{0}$ degeneration in anterior and medial parts of the colliculus, and after the medially $N$ situated damage in the left hemisphere of $N$ cat $66 \mathrm{~L}$ the degeneration is found more $\mathrm{N}$ posteriorly and laterally. The significance 
of this organization in terms of the representation of the retina upon area 17 and the superior colliculus will be discussed later; it may be noted that in none of these brains does the degeneration extend right up to the margins of the colliculus and this provides further evidence for the lesions being restricted to the cortex of area 17 , as such an extension to the margin is found only after the larger lesions which damage more than one area. Although the survival periods in these experiments varied between three and seven days no appreciable difference in the intensity or nature of the degeneration could be seen.

The observations in seven experiments in which the damage is confined to the cortex of area 18 are summarized in Fig. 5. All these lesions are in the anterior half of this area because its posterior half is largely buried within the walls of the postlateral sulcus, and all the lesions placed here have overlapped into adjoining areas. It is apparent, however, that there is also an organization in the projection of area 18 upon the colliculus. In the antero-posterior dimension it is similar to that found from area 17. All of the degeneration is in the lateral half of the colliculus, and when the damage is near the anterior limit of either area the degeneration is about the middle of the lateral margin; after more posteriorly placed lesions the degeneration occurs further anteriorly. Because area 18 is so narrow, it has not been possible to achieve such clear-cut results for the medio-lateral organization as in the case of area 17, but, despite this difficulty, there is a definite trend of difference in this dimension, and it is the reverse of that found for area 17. Thus, lesions close to the medial border of area 18 result in degeneration near the lateral edge of the colliculus and when the damage adjoins the lateral limit of this architectonic area the degeneration is found in a distinctly more medial position. It may be noted that this reversal
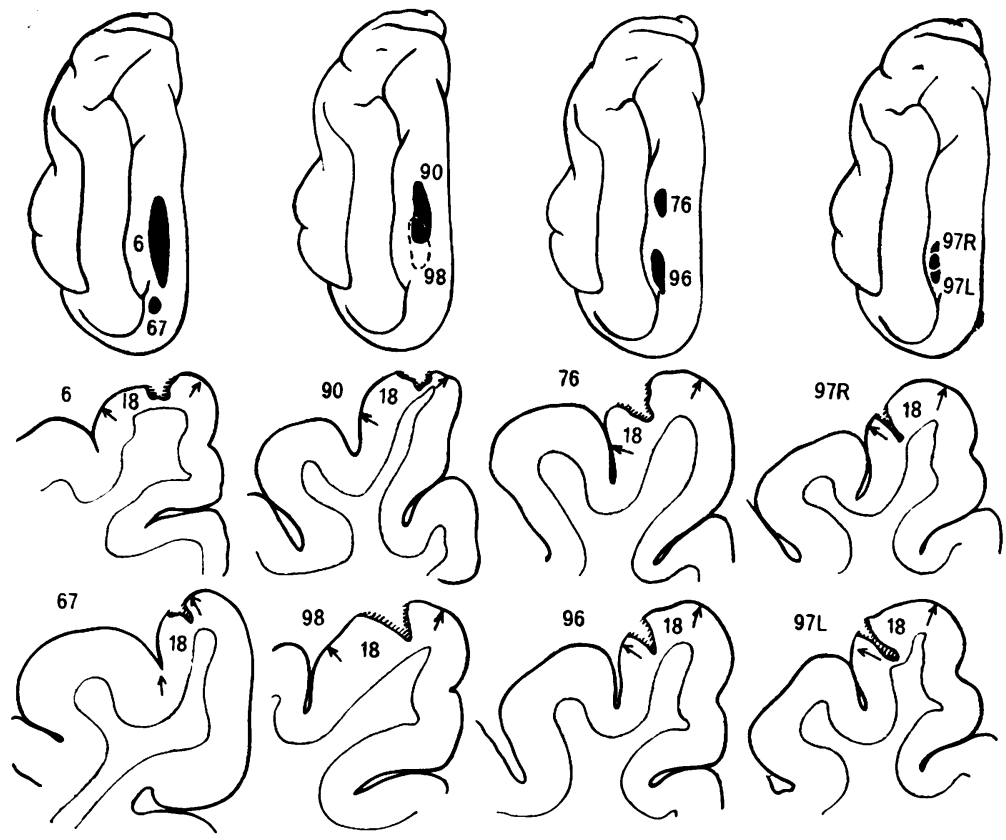

FIG. 5. To show the extent of the lesions which are confined to area 18, together with the distribution of the degeneration in the superior colliculus and in the lateral geniculate nucleus.
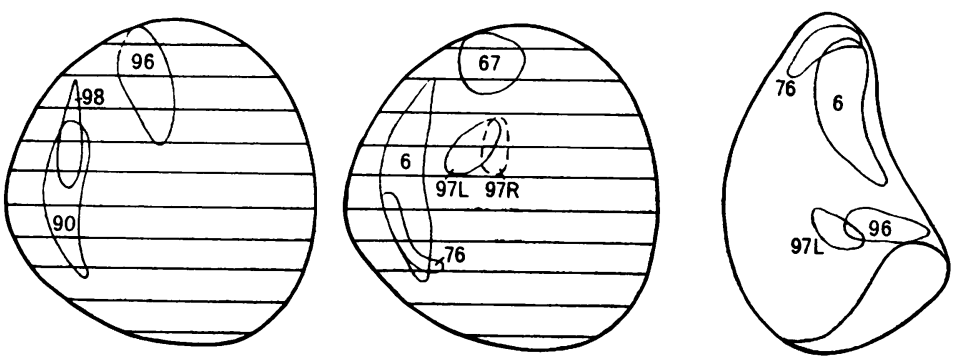


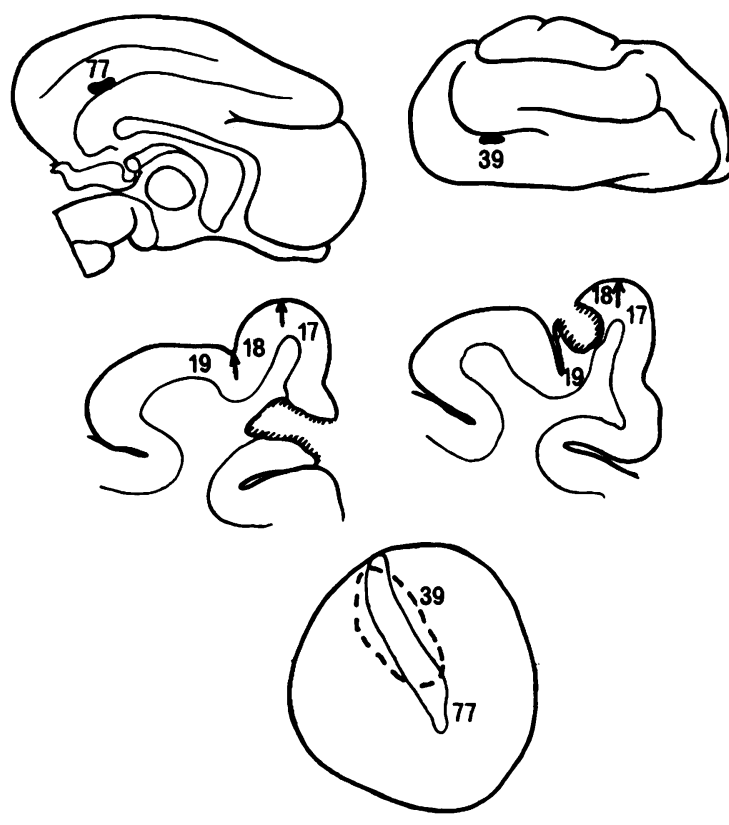

FIG. 6. The lesions and the degeneration in the colliculus in experiments $77 \mathrm{~L}$ and 39.

in the medio-lateral organization of the projection of area 18 upon the colliculus as compared with that of area 17 is in accord with the findings on the representation of the retina upon these areas. The position of the degeneration due to the relatively anteriorly-placed lesions in experiments 96 and 97 is somewhat surprising, as they overlap the representation of the horizontal meridian in the colliculus The explanation for this will be discussed separately in relation to recent findings on the representation of the horizontal meridian on the cortex. As in the group of experiments with selective damage to the cortex of area 17, the degeneration in the colliculus never reached one or other of its margins. The findings in all the experiments with large lesions are in accord with the organization already shown for areas 17 and 18, and only three further examples will be described in order to illustrate certain points regarding the interrelationship of those parts of the cortex and colliculus in which the horizontal meridian of the retina is represented.

In experiment 77L (Fig. 6) the cortex of the lower part of the splenial gyrus has been destroyed, and a slight extension into the white matter has almost certainly interrupted fibres from more dorsal parts of area 17 and possibly from the medial part of area 18. It is quite restricted in its antero-posterior extent and is immediately behind the level of the splenium of the corpus callosum. In the colliculus $\bar{Z}$ there is a relatively narrow band of degeneration $\stackrel{\mathbb{D}}{=}$ extending postero-medially from the antero-lateral 0 margin almost to the posterior edge; it is parallel $\stackrel{\text { ? }}{=}$ to, and slightly overlaps, the representation of the horizontal meridian of the retina. In another experiment, cat 39 , the area of degeneration in the $\delta$ colliculus is very similar in position and extent to that of $77 \mathrm{~L}$, although the lesion is more laterally placed, having destroyed the junction of areas $18 \stackrel{\Rightarrow}{\Rightarrow}$ and 19 in the medial wall of the lateral sulcus. $\stackrel{5}{9}$ As the areas of degeneration in the colliculus due 0 to these lesions at different medio-lateral positions $\frac{\bar{\sigma}}{\bar{\sigma}}$ in the cortex overlap each other and are all in the $\frac{\bar{\rho}}{\partial}$ region in which the horizontal meridian is repre- $\stackrel{\mathbb{}}{\propto}$ sented, it is clear that this part of the colliculus is related to both areas 17 and 18 . The fact that the degeneration in experiment $77 \mathrm{~L}$ does not reach the ${ }^{\circ}$ posterior margin of the colliculus is readily explained $\vec{\omega}$ on the basis that the lowermost part of area 17 has $\stackrel{5}{.}$ not been involved, but the absence of degeneration $\overline{3}$ in the posterior half of the colliculus in cat 39 after? destruction of the most lateral part of area 18 is $\omega$ surprising, although it is in agreement with certagin recent electrophysiological findings.

There is no experiment in which the lesion ${ }_{6}^{\circ} \mathrm{O}$ confined to area 19, and there is therefore no direets evidence that this area has an independent projection $\vec{\longrightarrow}$ to the superior colliculus. In five brains in whigho this area is involved in addition to one or both $8 \mathrm{f}=$ the other subdivisions of the visual cortex the sifeco of the degeneration in the colliculus is in accord witho the organization already described, and the laminar arrangement of the degeneration is similar to that in the earlier experiments, but there is probably more degeneration in the stratum intermediale and there is a suggestion that some of the fibres from $\propto$ the anterior part of area 19 enter the deep aspect $\Rightarrow$ of the colliculus from the cerebral peduncle as wello as through the brachia.

The following group of experiments, in which lesions were placed in 'non-visual' areas of the cortex, shows that the superior colliculus receives? fibres from most parts of the neocortex. In cats 69. and 64 (Fig. 7) the damage is in the upper and lower parts respectively of the posterior supra sylvian gyrus; degenerating axons are seen in the pretectum and brachia of the ipsilateral superior colliculus and terminal degeneration is present in the antero-medial quadrant of the colliculus; as after lesions of the visual cortex, the degeneration is found predominantly in the stratum opticum $N$ the ventral part of the stratum griseum super ficiale, and the dorsal part of the stratum griseum? intermediale. There is noticeably more degeneration 

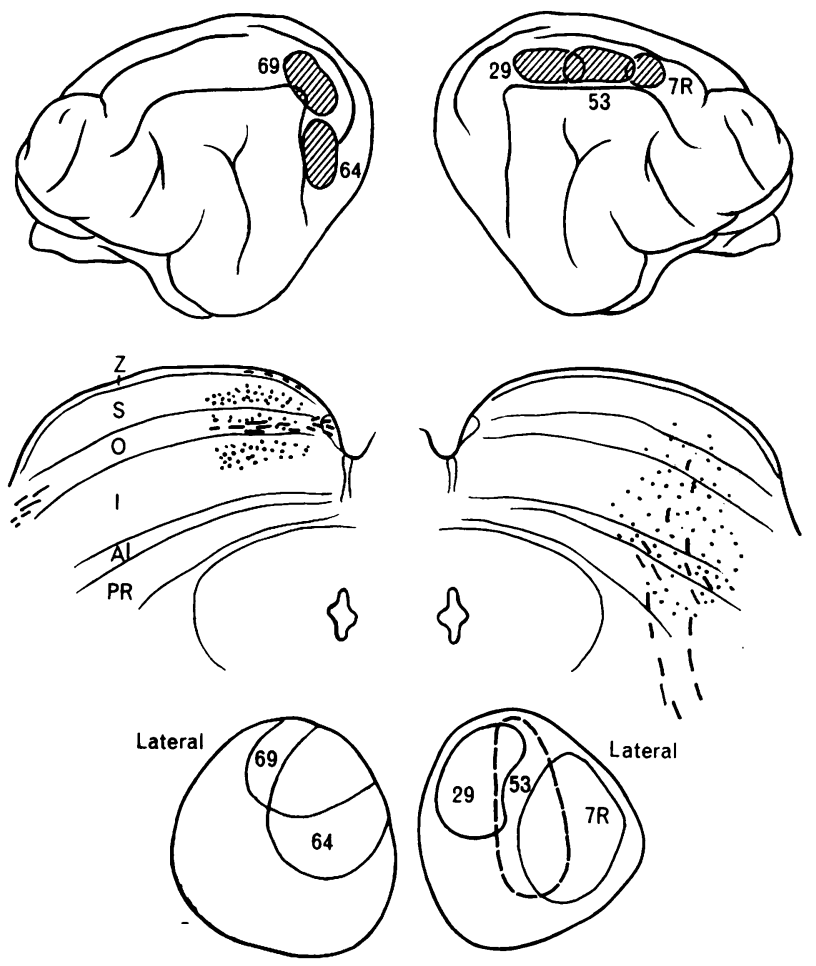

FIG. 7. The site of lesions in the suprasylvian gyrus and the resulting degeneration on coronal sections and on orthographic reconstructions of the dorsal surface of the superior colliculus. In this figure the lesions in the middle suprasylvian gyrus are shown as though on the right side in order to emphasize the difference in laminar distribution of the degeneration. It should be noted that degenerating fibres ascend to the colliculus from the cerebral peduncle only in experiments $7 R$ and 53, but the laminar distribution of the degeneration in these two experiments and in cat 29 is the same. in the stratum griseum intermediale, however, than after lesions of the visual cortex.

In three experiments lesions were placed in the middle suprasylvian gyrus (Fig. 7 ). In cat 29 , in which the damage is in the posterior third of the gyrus, degenerating fibres enter the colliculus only from the brachia, whereas in the other two experiments, in which the middle and anterior parts of the gyrus are involved, fragmented axons reach the colliculus laterally from the brachia and also ventrally from the midbrain tegmentum. The latter fibres can be traced back to the ipsilateral cerebral peduncle which they leave near the middle of the antero-posterior extent of the medial geniculate nucleus. Most of these fibres ascend between this nucleus and the substantia nigra, passing through the mesencephalic reticular formation, but some traverse the substantia nigra. The terminal degeneration in the colliculus of cat 29 is confined to the antero-medial part of its extent, that in experiment 53 forms a broad band in the middle of the anterior two-thirds, and in $7 \mathrm{R}$ it is more extensive and occupies the lateral half of the colliculus. The degeneration is present in several layers-in the strata opticum, griseum superficiale, griseum intermediale and profundum. A large lesion was placed in the upper part of the anterior suprasylvian gyrus in experiment 55L. Degenerating axons reach the superior colliculus by passing dorsally from the cerebral peduncle through the midbrain tegmentum, and the terminal degeneration is distributed in a manner similar to that in the previous group, but, in addition, some fragments are present in the dorsolateral part of the subjacent central grey.

In several experiments, some of which are available from other studies, the damage is situated in one or other subdivisions of the somatic and auditory sensory areas (Fig. 8). In three of these brains there are lesions in different parts of the auditory cortex, and four in which either the primary or secondary somatic sensory cortex has been damaged. In all of these brains a small number of degenerating fibres ascend dorsally from the ipsilateral cerebral peduncle and enter the ventrolateral aspect of the colliculus. Within the latter, terminal degeneration is found in the stratum griseum intermediale and profundum and there is also a little in the central grey. Even a very small lesion in the somatic sensory cortex results in a readily detectable focus of degeneration in the colliculus; the small area of damage in experiment 315 was caused by stabbing the cortex of the posterior 
bank of the coronal sulcus at the middle of its extent, and there is no involvement of white matter. Degenerating axons are clearly visible as they enter the ventro-lateral aspect of the colliculus, and they terminate in a small area in the lateral part of the stratum griseum profundum. Although the course of the degenerating fibres and the laminar distribution of the degeneration is essentially the same in all these brains, the topographical localization in the colliculus varies with the site of the lesion. After involvement of the auditory cortex the degeneration is limited to the posterior part of the colliculus, and, following lesions of the somatic sensory area, it is in posterior and lateral parts. Furthermore, when different parts of the primary somatic sensory area are damaged the site of the degeneration in the colliculus varies.

After destruction of the motor cortex in experiment 339 degenerating axons can be clearly seen leaving the cerebral peduncle to ascend to the antero-lateral part of the superior colliculus. Here they pass superficially, in a narrow band, as far as the stratum opticum, and terminal degeneration is present in the strata opticum, griseum intermediale and profundum, together with a little in the central grey.

DISCUSSION The finding of a substantial projection of the visual cortex upon the superior colliculus in the cat is in agreement with several other studies (Beresford, 1961; Altman, 1962; Sprague, 1963) and only certain main features of the course and termination of these fibres need be considered. The fibres from the visual cortex reach the superior colliculus through the pretectum and the brachia of the colliculus, and although a few of the fibres
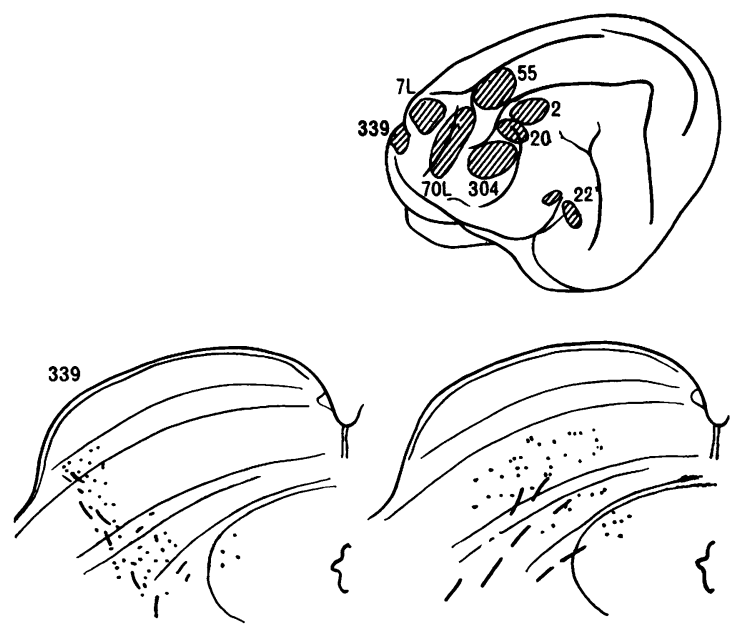

FIG. 8. To show examples of experiments with damage in the motor cortex (339), somatic $(7 L, 70 L, 304)$, and auditory $(2,20,22)$ sensory areas and in the anterior suprasylvian gyrus (55). The laminar distribution of the degeneration in the superior colliculus after these lesions is shown on coronal sections. ventral part of the stratum opticum from which they $Z$

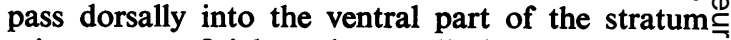
griseum superficiale and ventrally into the stratum griseum intermediale. The fibres thus enter the $\frac{c}{0}$ colliculus by the same pathway as those from the ${ }_{T}$ retina, terminate in essentially the same layers, and, as far as one can judge from the intensity of the $\frac{\rho}{-}$ degeneration, are of approximately the same number as those from the contralateral eye.

Whether these two groups of afferent fibres $\Rightarrow$ terminate upon the same cells, different cells, or $\stackrel{\text { p }}{+}$ different parts of the same cell remains to be을 determined, but the intensity of the degeneration $\frac{\bar{\sigma}}{\bar{s}}$ together with the similarity of the laminar distribu- $\frac{\vec{\sigma}}{\overrightarrow{0}}$ tion indicates a very close relationship. Despite $\stackrel{\mathbb{Q}}{2}$ these important similarities the retinal and cortical $\%$ projections upon the colliculus differ in certain $\vec{\circ}$ respects: first, the retinal projection is bilateral whereas the cortical projection is strictly unilateral; $\vec{\omega}$ secondly, the fibres from these two sources run at different levels within the stratum opticum, the retinal being superficial to the cortical, and finally, $i$ a few of the cortical fibres end in the stratum zonale $\vec{i}$ whereas none from the retina do so.

The experiments with small lesions have show $\vec{\phi}$ that there is a well-ordered arrangement in th: projection of the visual cortex upon the colliculug and also that at least two of the architectonic sub $\rightarrow$ divisions, areas 17 and 18, have an independegit projection to the colliculus. In the antero-posteriot dimension the organization is the same for bot these areas, anterior parts of each area being related to lateral parts of the colliculus and posterior parts medially. This finding by itself suggests that those parts of the cortex and colliculus related to a

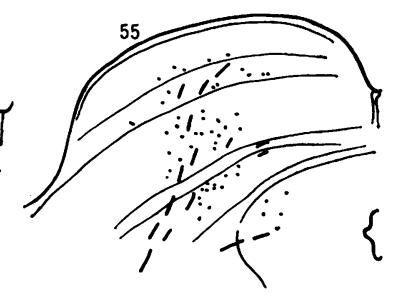


particular region of the retina are interconnected, as the superior retinal representation is found anteriorly in the cortex and laterally in the colliculus, whereas the inferior retina is represented posteriorly in the cortex and medially in the colliculus. That this relationship is also present in the medio-lateral dimension is shown by an analysis of the results of all the other experiments. As the organization in this dimension is different in areas 17 and 18, and also because there is a separate representation of the retina in each of these areas, they will be treated separately.

The vertical meridian is represented along the border of areas 17 and 18 and the peripheral margin of the retina along the medial, or inferior edge, of area 17; the superior and inferior ends of the meridian are respectively anterior and posterior, and the area centralis is at approximately the junction of the lateral and post-lateral sulci (Talbot and Marshall, 1941; Bilge et al., 1963; Hubel and Wiesel, 1965). In the superior colliculus the vertical meridian is represented along the anterior margin, the periphery of the retina on the posterior margin, with the superior and inferior ends of the meridian being respectively lateral and medial (Apter, 1945). If the site and extent of the lesions in the cortex of area 17, together with the resulting foci of degeneration in the colliculus, are considered in terms of these representations of the retina, it is clear that the projection of area 17 is organized so that parts of the cortex related to a particular part of the retina send efferent fibres to that part of the superior collicus which receives fibres directly from the same site in the retina. Thus damage of lateral parts of area 17 results in degeneration in anterior parts of the colliculus (Fig. 4), in both of which is the representation of the vertical meridian, and after lesions in the medial parts of area $\mathbf{1 7}$ degeneration is found posteriorly in the colliculus, to both of which the periphery of the retina is related. In a group of experiments with small lesions situated close to the lateral margin of area 17 , but at different antero-posterior levels, it can be seen that as one moves posteriorly on the cortex (from superior to inferior on the meridional representation) there is a progressive shift of the degeneration in a medial direction and parallel to the anterior margin (Fig. 4). The finding in the three experiments with small medial lesions shows a similar progression of the degeneration in a medial direction. The fact that there is no experiment with a restricted focus of degeneration close to the posterior margin of the colliculus is due to the difficulty in placing a lesion restricted to the superior bank of the splenial sulcus.

In regard to area 18 there are lesions limited to this subdivision only in the anterior half of its extent, and because of its limited width it has not been possible to provide such definite evidence for the organization in this dimension as for area 17. Comparison of the experiments illustrated in Fig. 5 shows that damage of the medial border of this area results in degeneration close to the lateral margin of the colliculus, and that as the damage becomes progressively more laterally situated the degeneration moves further medially. This projection of the medial part of area 18 to the lateral part of the colliculus again indicates that parts of the cortex are projecting to those parts of the colliculus which have the same retinal representation, as the vertical meridian is known to be related to each of these. The distribution of the degeneration in the middle of the colliculus after the more laterally situated lesions in area 18 , however, is surprising, as the horizontal meridian of the retina is known to project to this part of the colliculus. It may be noted that essentially similar observations were made in three separate hemispheres and after repeated examination. They are explicable on the basis of the recent findings of Bilge, Bingle, Seneviratne, and Whitteridge (1967) that the representation of the horizontal meridian forms the boundary between the functional areas, visual 11 and 111 (equivalent to areas 18 and 19). It would appear from our material on the anterior half of area 18 that most of this area is related to more central parts of the retina, and that the representation of the periphery is squeezed into its more anterior part, because degeneration was found to encroach upon this part of the retinal representation in the colliculus only after the lesions which extended most anteriorly $(6,76$, and 90$)$. This confirms the conclusions of Hubel and Wiesel (1965). That the entire width of areas 17 and 18 at the level of junction of the lateral and post-lateral sulci also project to the region of representation of the horizontal meridian in the colliculus is shown by the experiments illustrated in Fig. 6.

There are no experiments with damage restricted to area 19 or to the cortex of the walls of the lateral suprasylvian sulcus, and there is therefore no evidence that these areas also have an independent projection to the superficial layers of the colliculus. It is not possible, therefore, to make the generalization that the cortical areas which receive a direct projection from the lateral geniculate nucleus also project directly to those layers of the colliculus which receive fibres from the retina. The fact that area 19 projects back to the medial interlaminar nucleus of the lateral geniculate body independently of areas 17 and 18 would suggest that it also does so to the colliculus.

From a correlation of his own experimental findings on the projection of the visual cortex upon 
the superior colliculus in the rat with previously published maps of the retinal representation in these areas, Lund (1966) found an organization in this projection comparable to that in the present study. $\mathrm{He}$ also noted that lesions in the antero-lateral part of the visual area resulted in relatively large areas of degeneration in the colliculus, but this has not been an obvious feature in our material. Lund found that anterior to the visual cortex there was a second area, lesions of which resulted in some fibres passing more caudally in the cerebral peduncle and terminating more deeply in the colliculus. Whether the projections from the two subdivisions of this area are homologous with the independent projection from area 18 or with the projection from the extra-visual cortex cannot be decided, but the more caudal course and deeper termination would support the latter possibility.

That areas of the neocortex outside the striate or peristriate cortex project upon the superior colliculus is well known (see Kuypers and Lawrence (1967) for references) but most reports of such connexions have been isolated instances concerned with particular areas of the neocortex. Recent, more comprehensive studies have shown that in both the cat (Sprague, 1963) and monkey(Kuypersand Lawrence, 1967) most of the neocortex projects upon the colliculus. Our material shows that in general terms this projection may be subdivided, in that the posterior and middle suprasylvian gyri project upon the same superficial layers and through the same pathway as does the cortex of areas 17 and 18, but at the junction of the anterior and middle suprasylvian gyri a change occurs in that the fibres pass more caudally in the cerebral peduncle, enter the deep aspect of the colliculus, and end in deeper layers. All functional cortical areas participate in this projection, and some organization is indicated by the present findings in the cat and by those in the monkey (Kuypers and Lawrence, 1967); the somatic sensory cortex is related only to the posterior and lateral parts of these layers, the auditory cortex to posterior parts, and the motor cortex to anterolateral parts. Even small, discrete lesions in these areas result in easily detectable foci of degeneration, and the position of this degeneration varies according to the site of the damage.

The change in laminar distribution of the degeneration and in the course taken by the cortico-tectal fibres occurs near the anterior end of the middle suprasylvian gyrus, and the cortex of the middle and posterior suprasylvian gyri is similar to that of areas 17 and 18 not only in the course and termination of their cortico-tectal fibres but also in having a comparable degree of organization in the anteroposterior dimension. This similarity probably results from the presence in this part of the gyruso of area 19 and the lateral suprasylvian area. Each $z$ of these areas receives a direct projection from the lateral geniculate nucleus, is interconnected with the other visual areas by association and callosa $E$ fibres, and may be expected, therefore, to project to the same layers in the colliculus as does thes retina. In the monkey a similar pattern has been shown (Kuypers and Lawrence, 1967) in thate occipito-temporal areas of cortex all project to superficial layers of the colliculus; these include $=$ area 17 , which is in receipt of geniculate efferent? fibres, and also areas 18 and 19 and temporale cortex, which receive association fibres from areaㅡㅡㄹ 17. In this context it is of interest that the somatic sensory cortex is sending fibres to the deep layerso of the lateral part of the superior colliculus whiches receives fibres from the dorsal column nuclei andspino-tectal tract (Nauta and Kuypers, 1958; Mehler, 1962; Kuypers and Lawrence, 1967), and $\vec{\omega}$ from which responses from the proprioceptors in eye muscles have been recorded (Fillenz, 1955; Cooper, Daniel, and Whitteridge, 1955). Furthermore, as far as can be determined from the brief description in the literature of the termination dn? the colliculus of ascending fibres in the auditopycu pathway (Woolard and Harpman, 1940; Moore afdo Goldberg, 1963, 1966) the same relationship ifs? present between the cortical and ascending fibres in this pathway. Thus, the visual, somatic, and으․ auditory areas of cortex appear to send fibres to the superior colliculus and these enter and terminates in the same way as do fibres from more peripheral. levels of the same sensory system (see Fig. 13).

\section{CORTICO-CORTICAL CONNEXIONS}

RESULTS The material is not suitable for a system- $\frac{\mathbb{Q}}{\mathbb{Q}}$ atic analysis of the association and callosal con- $\overrightarrow{\vec{F}}$ nexions of each of the architectonic or functionalo subdivisions, either because the damage involvedmore than one of these areas in brains with unilateralo lesions, or because the experiments with small lesions confined to one of these areas were frequently accompanied by another on the opposite side.However, as the published evidence on the cortical 3 connexions of the visual area is meagre and some-i what discordant, certain of our observations will be 3 described.

In two experiments in which most of the visual? cortex was removed on one side there is degeneration? throughout the antero-posterior extent of the visual cortex on the contralateral side, but its medio-lateral distribution varies considerably at different levels $N$ (Fig. 9). There is heavy degeneration in area 18, and over most of the area it is limited to approximately 

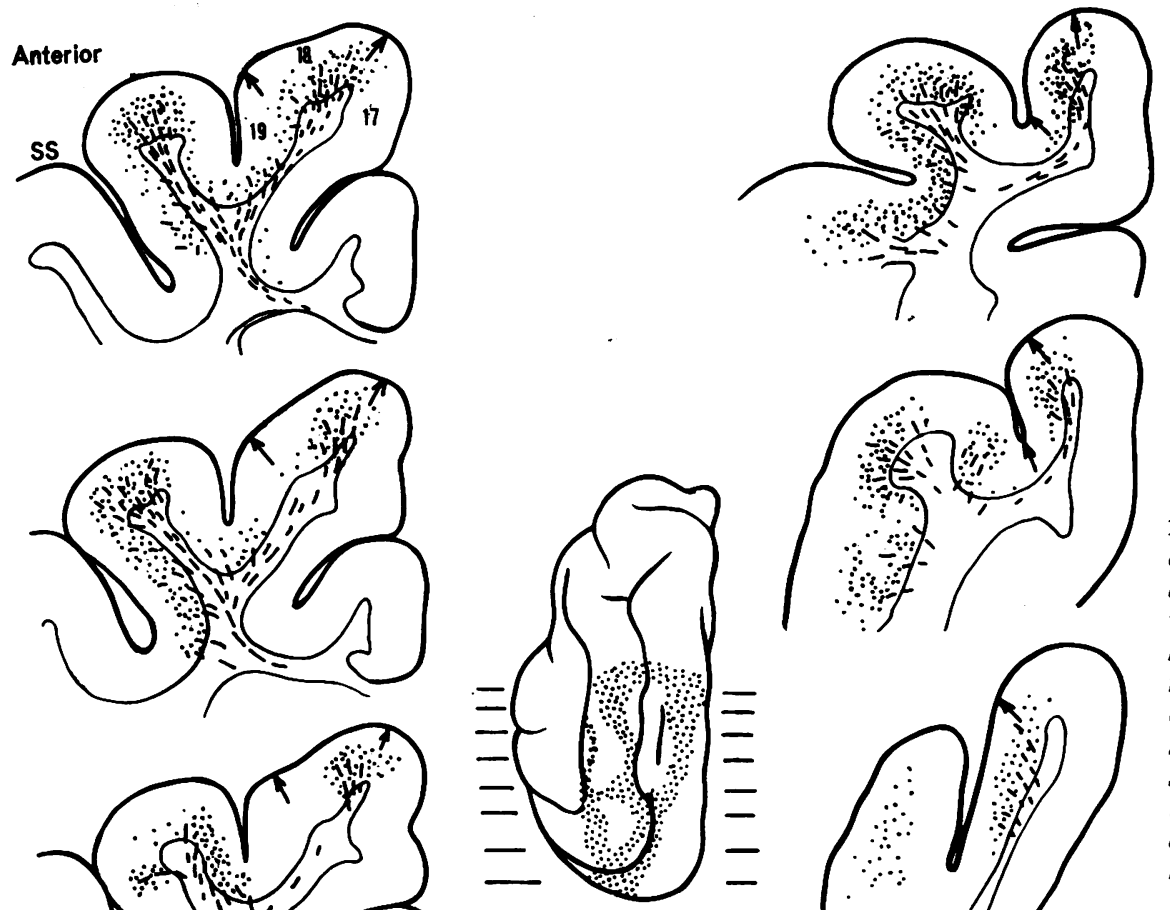

FIG. 9. The distribution of the terminal degeneration in the visual cortex of the left hemisphere following

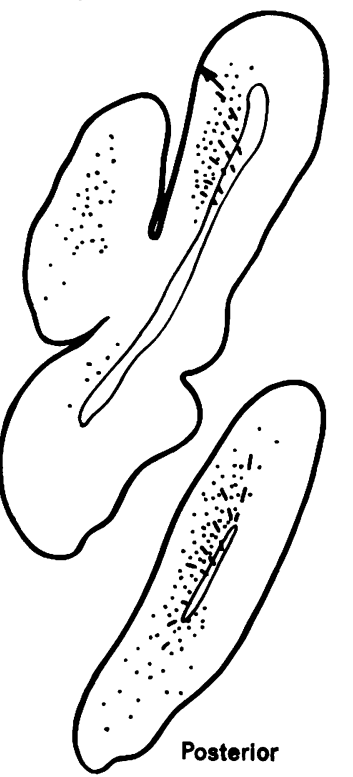
removal of the visual cortex of the opposite side. The levels of the sections are indicated on the drawing of the dorsal surface of the hemisphere.

the medial half. The degeneration in the medial part of area 18 definitely extends into the adjoining area 17, but only the extreme lateral part of the latter is involved and the density of the degeneration is much less, the diminution occurring at the border of areas 17 and 18. This degeneration in area 17 extends caudally right on to the posterior surface of the hemisphere, thus almost certainly behind the level of area 18. The rest of the medio-lateral extent of area 17 is completely clear of any fragmentation. Except at the middle of its antero-posterior extent the degeneration in area 18 is bounded laterally by a clear area; anteriorly, this area occupies the lateral part of the dorsal surface of the lateral gyrus and the medial wall of the lateral sulcus, but more posteriorly it is largely within the walls of the sulcus. The clear area is in turn delimited laterally by a band of degeneration in area 19. At the junction of the lateral and post-lateral sulci this degeneration again fills the whole width of area 19 and is continuous with that in area 18 . In between, however, it affects only part of the width of area 19; over most of the antero-posterior extent, the lateral half of this area is involved, but at the anterior and posterior ends more medial parts are affected. On sections through the anterior part of the visual area this 
degeneration in area 19 is in the walls of the lateral sulcus, but more posteriorly it emerges onto the surface of the posterior suprasylvian gyrus. In that part of area 19 situated in the depth of the splenial sulcus there is only an occasional fragmented fibre. There is also severe degeneration in the medial wall or floor of the middle suprasylvian sulcus throughout its antero-posterior extent. Between and parallel to the bands of degeneration in area 19 and in the floor of the middle suprasylvian sulcus there is a further narrow band of degeneration in the cortex of the exposed dorsal surface of the suprasylvian gyrus, and this is bounded medially and laterally by relatively clear zones (but this is due to the destruction of the symmetrical region). In terms of the architectonic areas the total callosal degeneration would appear to be in the extreme lateral part of area 17 , the medical part of area 18, the lateral part area 19 (with only a little in the part of this area in the splenial sulcus) and the cortex of the dorsal and lateral parts of the middle suprasylvian gyrus. At approximately the middle of its antero-posterior extent the degeneration forms a continuous mediolateral band (Fig. 9).

Although the laminar arrangement of the degenerating fibres and terminal degeneration within the cortex is essentially the same in all these areas, there are distinct differences in the intensity and orientation of the fibres as they enter the cortex. Degenerating fibres are seen in all layers, but they are most numerous in layers 5 and 6 , become progressively less towards the surface, and only an occasional one is seen in layers 1 and 2 . Fine, preterminal degeneration is clearly most intense in the deeper part of layer 3 and throughout layer 4, with very little in the other layers. The degeneration is severe in area 18 and the lateral suprasylvian cortex, and there is appreciably less in areas 17 and 19. In areas $\mathbf{1 7}$ and 18 the majority of the incoming fibres tend to be orientated perpendicular to the surface, with only occasional ones running obliquely, whereas those which enter area 19 and the lateral suprasylvian cortex do so very obliquely.

Several experiments with small lesions confined to the cortex of area 17 show that this area sends association fibres to quite separate foci in areas 18, 19 , and the lateral suprasylvian cortex; in area 17 itself there is degeneration for a short distance on either side of the lesion, but not in a separate focus (Fig. 10). The degenerating fibres leaving the damaged area of cortex are clearly running perpendicular and this is in contrast to the obliquity of those entering the other areas on the same side. In three experiments in which the damage is restricted to the medial part of area 18 association fibres can be traced to the lateral half of area 17, to area 19, and again to the $\stackrel{\mathbb{C}}{\stackrel{\circ}{\circ}}$ lateral suprasylvian cortex. The intensity of degen- $\frac{\text { o }}{Z}$ eration in all these brains is always heaviest in $\mathbb{Q}$ area 18 and in the lateral suprasylvian cortex, and $\frac{c}{\circ}$ least in areas 17 and 19 and the dorsal supra-
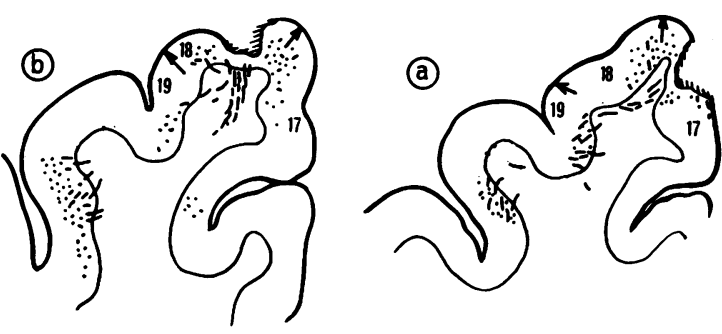

10. The distribution of the degeneration in adjoining areas of the visual cortex following damage restricted to area $17(a)$ and to area $18(b)$.

sylvian cortex. These degenerating association $\omega$ fibres are found over a considerable antero-posteriow $\vec{i}$ extent, and whether this is related to the represent tion of particular parts of the retina cannot definitely stated. The distribution of the degeneration in the different areas varied in the medio-laterai dimension depending upon the precise site of the lesion in area 17.

In area 18 the distribution of the degenerations association fibres has a very striking pattern, ata after large lesions they have been found to extend for considerable distances away from the lesions. While there is a little fragmentation in all layers of the cortex it is much more pronounced in layer 4 and the deep part of layer 5; the fragments here are $\frac{O}{\mathbb{D}}$ arranged in a tangential plexus, and stand out $\varrho$ conspicuously as two bands corresponding to the $\overrightarrow{\overrightarrow{0}}$ outer and inner bands of Baillarger. As this arrange- 3 ment is found equally clearly at points well away? from the damaged area, it suggests that the associa-? tion fibres to and within this area are running mainly intracortically. In area 19 and the lateralo suprasylvian cortex the degenerating fibres enter:and run very obliquely in layers 5 and 6 , the fine terminal degeneration is predominantly in layer $4 \%$ and superficial to this there is very little.

There is no experiment in which the damage iso restricted to either area 19 or the lateral supra- $\rightarrow$ sylvian cortex, so nothing can be said about the efferent cortical connexions of these areas. Most of the association and callosal connexions which have been described so far terminate in areas which $\tilde{O}$ 
are known to receive a direct projection from the lateral geniculate nucleus. That the visual cortex does project to other cortical areas, however, is shown by the finding of a localized focus of degeneration in the cortex of area 6 on the medial part of the anterior sigmoid gyrus after large lesions of the lateral gyrus involving areas 17, 18 and 19 and also after smaller lesions virtually confined to area 17 or 18.

DISCUSSION The distribution of the degeneration in the visual cortex of the opposite hemisphere found in the present experiments after interruption of the callosal fibres is in good agreement with the description of similar experiments by Ebner and Myers (1965), but our interpretation of this pattern in terms of the architectonic subdivisions is different, and we shall attempt to correlate it with the functional representation of the retina in the cortex. Ebner and Myers (1965) considered that area 17 was completely free of degeneration, that most of the degeneration was in an area 'corresponding closely to area 18' and that lateral to this was a degeneration-free zone which was analogous to, but not closely corresponding to, area 19. In our opinion, although most of area 17 is undoubtedly free of degeneration, its extreme lateral part does contain degeneration; only the medial half or so of area 18 is affected, its lateral half and the adjacent medial part of area 19 , over most of their antero-posterior extent, remain free; it is mainly the lateral part of area 19 which contains degeneration. In addition, there is degeneration in the walls of the lateral suprasylvian sulcus. Because only the extreme lateral part of area 17 is affected, it might be argued that this is largely a question of precise definition of the boundarynonetheless, we are convinced that the degeneration does extend medial to the pyramidal cells of the third layer of area 18, that at the junction of areas 17 and 18 there is a distinct change in the intensity of the degeneration, and finally that the degeneration extends to the extreme caudal surface of the hemisphere which is behind area 18. The width of the degeneration in area 18 varies along its anteroposterior extent, being widest at the junction of the lateral and post-lateral sulci, where it involves the entire medio-lateral extent and is continuous with that in area 19. It is difficult to escape the conclusion that the lateral band of degeneration is in area 19, and the progressively more lateral disposition of this degeneration at more posterior levels fits with this interpretation; furthermore, if this band of callosal degeneration is not in area 19 it would be necessary to conclude that area 19 does not receive association fibres from area 17 and is not equivalent to the third functional visual area, because the region receiving the callosal fibres also receives association fibres from area 17 and is within that mapped as visual 111 by Hubel and Wiesel (1965).

It has been suggested that the callosal connexions connect those "cells of the visual cortex whose receptive fields are closely adjacent to the vertical meridian' of the retina (Choudhury, Whitteridge, and Wilson, 1965) and to a certain extent our findings would support this. In the first place, the vertical meridian of the retina is known to project to the border between areas 17 and 18 , a region which is rich in callosal connexions; secondly, in area 18 the peripheral representation of the retina is displaced rostrally and posteriorly (Hubel and Wiesel, 1965), and the representation of the horizontal meridian comes to occupy the junction of areas 18 and 19 (Bilge et al., 1967), and it is significant that a considerable portion of this junction is free of callosal degeneration. The representation of the vertical meridian in area 19 is along its lateral edge and this part does receive callosal fibres. The level at which commissural degeneration fills the entire width of areas 18 and 19 can be interpreted as being the expanded representation of the area centralis, and where the bands of degeneration approach each other at their anterior and posterior ends are the representations of the superior and inferior ends of the meridian. On the other hand, there are certain difficulties about this interpretation, and foremost is the fact that the extent of the degeneration in the lateral part of area 17 must certainly be less than the representation of the vertical meridian in this area; whether this lateral part of area 17 should be considered as a separate transitional area, functionally distinct from the rest of area 17 and possibly homologous with that described in primates by Hassler (1966) or related to the narrow zone of overlap in the retina described by Stone (1966) must remain speculative. The interpretation of the pattern of callosal connexions put forward here for the cat can also be applied to the description of these connexions in the monkey (Myers, 1962). It would appear that in the monkey, following occipital lobectomy, the extreme edge of the opposite striate cortex, in which the vertical meridian is represented, contains degeneration. Furthermore, the whole width of areas 18 and 19 is not filled, except at the representation of the macula, and there is a belt free of degeneration in the adjoining parts of areas 18 and 19. The possibility that the callosal fibres connect those parts of the cortex related to the representation of a part of the retina and not complete architectonic or functional areas is indirectly supported by the findings on the somatic sensory 
cortex. Ebner and Myers (1965) pointed out that in the primary somatic sensory area (of the cat) the forelimb and distal hindlimb areas are free of degenerating fibres, while the cortical regions related to the tail, proximal hindlimb, trunk, and face are connected by the corpus callosum. These observations have been extended and confirmed in a detailed analysis of the somatic sensory cortex in the cat and monkey (Jones, 1967), where it has been found that in both the first and second areas the callosal connexions are between the same functional areas mentioned by Ebner and Myers (1965) and are not between any particular architectonic area. The additional finding by Jones that in the somatic sensory area the areas which do not receive commisural connexions also do not send any callosal fibres raises the possibility that the same may be true of the visual cortex. The two experiments illustrated by Hubel and Wiesel (1965) as lesions of area 17 which resulted in degeneration in the contralateral hemisphere could well have interrupted fibres from the junction of areas 17 and 18, but, until there is more evidence from experiments with small lesions in the visual cortex, one must be cautious about the origin of callosal fibres, and this is especially so in regard to those terminating in the dorsal suprasylvian cortex.

Our material for the study of the association connexions of the individual architectonic subdivisions is limited, but it confirms the results of Hubel and Wiesel (1965) that area 17 projects to areas 18, 19, and the lateral suprasylvian cortex, and it also shows that area 18 sends fibres back into area 17, and again to area 19, and the cortex of the middle suprasylvian sulcus. The medio-lateral distribution of the degeneration in these areas varies with the medio-lateral disposition of the damage, and this indicates that a more detailed analysis would be of value. It is noteworthy that the antero-posterior spread of the association fibres is much more extensive than that of the callosal fibres (the latter are essentially limited to an area symmetrical to the extent of the damage). The association fibre connexions of area 17 with areas 18 and 19, and of 18 with 17 and 19 in the cat, are very similar to the findings of Kuypers, Szwarcbart, Mishkin, and Rosvold (1965) in the monkey, but it is difficult to be certain that there is a homologue of the lateral suprasylvian cortex in the latter, although their parieto-occipital area might be this as it receives a few association fibres from area 17, many from areas 18 and 19, and callosal fibres from the visual cortex of the other hemispheres. A surprising finding is that the majority of the cortical connexions -association and callosal-of the visual area in the cat are between areas of cortex which receive a direct projection from the lateral geniculate nucleus. 으 There would appear to be few connexions to or from $Z$ 'association' areas of cortex. There is also a definite $\stackrel{\mathbb{D}}{\subseteq}$ connexion between the visual area and a localized part of area 6 in the medial part of the anterior sigmoid gyrus. This projection was found not only after large lesions of the visual cortex, but also when the damage was essentially confined to area 17 , and $\frac{\rho}{5}$ this is again in agreement with the findings in the monkey (Kuypers et al., 1965). Finally, the possibility of a small connexion with area 7 cannot be excluded, $\vec{\Rightarrow}$ because it was not possible to be certain of the $\stackrel{?}{?}$ precise boundary between this area and the anterior limits of area 19 and the suprasylvian areas.

\section{OTHER SUBCORTICAL PROJECTIONS}

RESULTS Dorsal lateral geniculate nucleus In all experiments in which the cortex of the lateral gyrus was damaged, degeneration is found in the dorsal $\vec{\omega}$ lateral geniculate nucleus. Experiment 1, with a $S$ relatively large lesion of areas 17 and 18, which was used to illustrate the main features of the cortico-tectal projection will be referred to again In the coronal sections of this brain (Fig. 1) the $i$ majority of the fibres passing dorsal to the latera $\vec{\omega}$ geniculate nucleus are orientated in a medio-laters direction, and the degenerated fibres are arrangegt likewise, dispersed among the normal ones. Some $\rightarrow$ fibres, both normal and degenerated, are clear $B$ 음 passing at right angles to these, and enter the underlying lamina $A$ of the lateral geniculate nucleus. At the medial margin of the nucleus $:$ narrow band of degenerating fibres descends close $\square$ to the medial margin of the main laminae, and from this fibres pass into the medial parts of the ventral laminae. In the lateral geniculate nucleus coarse and fine degenerating fragments of fibres and of $\frac{\propto}{\square}$ pericellular plexuses are found in the medial parts $\overrightarrow{\vec{O}}$ of the main laminae over approximately the middle $\frac{0}{3}$ half of their antero-posterior extent. This degeneration is heaviest in lamina $\mathbf{A}$ and becomes progressively less dense in the more ventral layers, so that in lamina B there is only an occasional fragment. Comparison of the more lateral, unaffected parts? of the laminae with the medial ends, however, 3 indicates, that this is quite definite evidence of degeneration in these ventral laminae. The central $\frac{0}{3}$ interlaminar nucleus is affected as well as the main laminae, and there is no abrupt change, either in the density or the orientation of the degeneration, at $\frac{D}{0}$ the borders of the different laminae as is seen after lesions of the retina. The medial interlaminar $N$ nucleus is free of degeneration, and the bundle of $N$ degenerating fibres descending between it and the $N$ main laminae does not distribute to it. 
In the two brains with large lesions of the visual cortex which were cut in the sagittal plane, cats 78 and 311 , there is a band of degeneration in approximately the middle of the antero-posterior extent of the lateral geniculate nucleus. This band is clearly orientated at right angles to the plane of the laminae, from antero-superior to postero-inferior (Fig. 2). The density of the degeneration is greatest in lamina $A$ and progressively diminishes to lamina $B$ in which there is only an occasional fragmented fibre. Although the fibres are also predominantly arranged perpendicular to the laminae, this is not so clear as after retinal lesions, and the difference in the arrangement of the degeneration in the main laminae and the central interlaminar nucleus is less marked. Near the upper margin of lamina A, the fibres appear to follow one of two alternative courses. The majority are orientated in an antero-superior direction but other fascicles can be clearly seen turning upwards and backwards, and the latter are finer and more prominent near the posterior limit of the degenerated band.

The site and extent of the degeneration in the lateral geniculate nucleus varies with the position and size of the cortical damage, and after smaller lesions is sharply localized. It is invariably found in that part of the nucleus, in both the anteroposterior and medio-lateral dimension, which is known to project to the damaged area of the cortex (Figs. 4 and 5). Degeneration is present following damage restricted to either area 17 or area 18 , and after lesions restricted to the latter area the intensity of the pericellular degeneration is somewhat greater in the central interlaminar nucleus than in the adjoining main lamina. Although degenerating fibres coursing down the medial aspect of the main laminae are present in the majority of the experiments, unequivocal terminal degeneration in the medial interlaminar nucleus is found only in the five brains in which the lesion involves area 19. In some of these experiments degenerating fibres also pass down the medial side of the medial interlaminar nucleus, so that the latter is clearly outlined by two vertical bands of fragmented fibres. The cortical projection to this element is also topically organized, as degeneration is present in its anterior part after anterior lesions, and posteriorly after damage to the caudal part of the visual cortex. The distribution of the degeneration in the nucleus also varies in the dorsoventral dimension, but it has not proved possible to determine the organization in this plane.

Because of this topical arrangement of the degeneration and also because Nissl-stained sections show that retrograde cellular degeneration is present at seven days and occupies precisely the same position, it was necessary to try and eliminate the possibility that the fibre degeneration was retrograde in nature by using shorter survival periods. After five and six days there is little difference in the appearance of the degeneration, apart from a slight diminution in the number of coarse fragments. In six animals with survival periods of three days, and

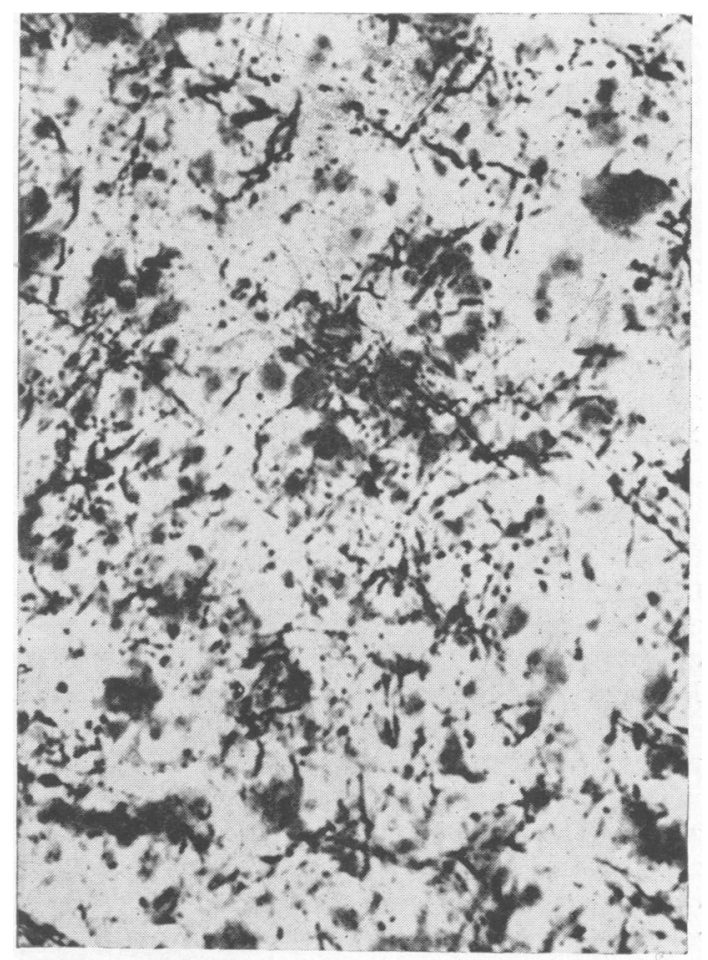

FIG. 11. Photomicrograph of degeneration in lamina $A$ of the lateral geniculate nucleus three days after damage of the visual cortex. $\times 640$.

with lesions of varying size, however, there is a distinct difference. The density of the degeneration in the affected parts of the nucleus is very much reduced, the size of the fragments is considerably smaller and they are more uniform; the pattern of degeneration is typically pericellular with less suggestion of a perpendicular orientation, and the difference in density between the different laminae is not so marked (Fig. 11). It may be noted, also, that the degenerating fibres which turn ventrally and laterally in the radiation to enter the nucleus are finer than the medially-directed coarse fibres, and also that the reduced density of degeneration in the geniculate is in no sense a technical deficiency 


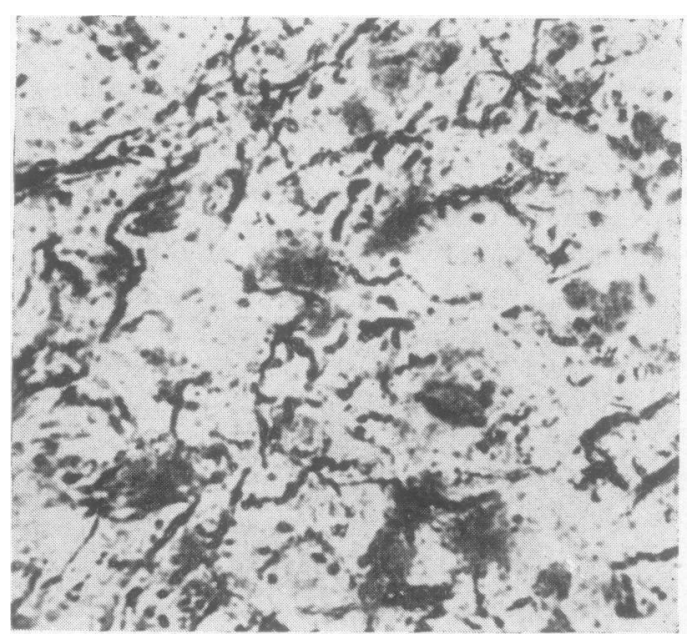

FIG. 12. Degeneration in the nucleus lateralis posterior of the thalamus three days after a lesion of the visual cortex. $\times 710$.

as the degeneration in the adjoining posterior nucleus, the nucleus lateralis posterior (Fig. 12), and the ventral lateral geniculate nucleus is as dense, or denser, than after the longer survival periods. In one respect, however, the degeneration after short survival periods resembles that in the other experiments, for it always affects those parts of the nucleus which are known to project to the damaged areas of cortex. Similarly, degeneration is seen in the medial interlaminar nucleus after three days only when the lesion has involved area 19 , but whether the fine, pericellular degeneration in the main laminae and central interlaminar nucleus at this short survival period is likewise related to area 17 or area 18 cannot be stated with certainty. The findings in one brain, cat 98, in which the damage is in area 17 on one side and in area 18 on the other suggests that this is so, as the degeneration is more intense around the cells of the central interlaminar nucleus than in the main laminae on the side of the area 18 damage.

Ventral lateral geniculate nucleus In the brains with relatively large lesions of the visual cortex, definite degeneration of medium sized fibres is present in the medial part of this nucleus. The degenerating fibres reach the nucleus by passing down on the medial aspect of the main laminae of the dorsal nucleus. Such degeneration is also found after survival periods of three days. It has not been possible to determine whether these fibres come from one or other of the architectonic subdivisions, as both the size of the lesions and the amount of resulting degeneration are too small to be certain.

Other thalamic nuclei In all the brains with lesions of the visual cortex terminal degeneration is found in certain other thalamic nuclei adjoining theō lateral geniculate nucleus. The terminology of this? region is confused, and for convenience we shalle base our description on that used and illustrated by Jasper and Ajmone-Marsan (1954). Following alא lesions of the visual area, irrespective of the size or site of the lesion, definite terminal degeneration is present in the nucleus lateralis posterior. The distribution of this degeneration varied in differentes? experiments, indicating that the cortical projectiono is an organized one; although a systematic corre-음 lation has not been made, it would appear thats anterior parts of the cortex project to anterior parts of the nucleus and medial parts to lateral parts. Then possibility that this fragmentation is retrograde in nature is largely obviated by the fact that it is present after survival periods of three days (Fig. 12), $\vec{\omega}$ and at this survival period it is much more intenseo than in the geniculate nucleus. Areas 17 and 185 each send fibres to this nucleus, as foci of degenera? tion are found in those brains in which the damage $\omega$ is restricted to one or other of these architecturar areas, and after minute lesions, as in experiment 32\% $\vec{\omega}$ When the damage is undoubtedly limited to these areas, the nucleus lateralis posterior has been only additional main thalamic nucleus to contin degeneration, but in larger lesions with involvemento of white matter, but still interrupting only fibes:from the visual cortex, degeneration is also fout in the nucleus labelled 'posterior' by Jasper arịto Ajmone-Marsan (1954), and lying immediatelyo adjacent to the medial interlaminar nucleus. It is however, quite separate from the latter, as degenera tion in the medial interlaminar nucleus can be seem to be separated by a clear zone from the affectedo medial part of the posterior nucleus. The precise site of origin of the fibres to this nucleus has noe been defined, but as such degeneration has been found only after deep white matter involvement i is probable that they arise from the medial part of area 17 or that part of area 19 in the superior bank of the splenial sulcus. After larger lesions whichp include area 19 there is also a little, scattereक terminal degeneration in the pulvinar and possibly. in the suprageniculate nucleus. It is not possible to be certain about the latter or to differentiate it from the medial part of the lateralis posterior Whether or not any of the degeneration involvess the posterior group of thalamic nuclei (Rose and. Woolsey, 1958) will be discussed later.

Following the larger lesions of the visual cortex terminal degeneration is found among the cells of the postero-dorsal part of the reticular nucleus. 
Striatum Fine pericellular degeneration is present in the body or tail of the caudate nucleus in most of the brains.

Nucleus of the optic tract, and accessory nuclei After large lesions there is a moderate degree of fine degeneration among the cells of the nucleus of the optic tract, and to a lesser degree in the pretectum medial to it. Although occasional scattered degeneration is seen in these areas after smaller lesions, it was not possible to analyse this in any detail, partly because of the minimal amount of degeneration and partly because there are fibres of passage through this region. After large lesions of the visual cortex a small amount of fine fibre and terminal degeneration is present in the accessory terminal nuclei, and is most clear in the lateral of these nuclei.

Pontine nuclei Definite terminal degeneration is found in those parts of the peduncular and lateral pontine nuclei immediately adjoining the basis pedunculi. It is present throughout the rostral halves of these nuclei and is predominantly unilateral, but after large lesions a little is seen in the corresponding nuclei on the contralateral side. In two brains with lesions almost certainly restricted to area 17 unequivocal degeneration is present in these nuclei on the ipsilateral side.

DISCUSSION The finding of marked degeneration in the dorsal nucleus of the lateral geniculate body five to seven days after damage of the visual cortex might at first be accepted as evidence for a corticogeniculate pathway, as these are the customary survival periods used for the Nauta technique (Beresford, 1961; Altman, 1962). However, in a system where reciprocal connexions may exist, where the distribution of the degeneration is in accord with the known organization of these connexions, and where cellular degeneration is already present at these survival periods, this fragmentation could well be retrograde fibre and dendritic break-up (Guillery, 1959; Cragg, 1962; Powell and Cowan, 1964). That fibre degenerationsparser, finer, and pericellular in arrangement-is also present at three days increases in probability that such degeneration is orthograde, but by no means makes it certain. Electron microscopic studies are critical in this regard, and therefore the illustration of degenerating presynaptic endings in the lateral geniculate nucleus after damage of the visual cortex is important (Szentágothai, Hámori, and Tömböl, 1966; our own unpublished observations, 1967). With the above reservations, the findings in the present study may be taken to support the electrophysiological evidence (Widén and AjmoneMarsan, 1960) for cortico-geniculate connexions.
If the fibre degeneration in the lateral geniculate nucleus is accepted as being orthograde in nature certain points may be commented upon. There is the possibility that the cortico-geniculate fibres may be distinguished from the geniculo-cortical on the basis of their size and number. In view of the fact that the fibre and terminal degeneration in the nucleus lateralis posterior of the thalamus and the superior colliculus is intense at three days, the question arises whether the degeneration found in the lateral geniculate nucleus is that of direct cortico-geniculate fibres or of collaterals of fibres to one or other of these other sites-especially the superior colliculus where there can be little doubt that the degenerating axons arise in the cortex. The cortico-geniculate projection appears to be topographically organized in the same manner as the geniculo-cortical as degeneration is invariably present in those parts of the nucleus known from previous studies to project upon the damaged area (Garey and Powell, 1967). Whether this is also true of the relationship between the individual architectonic areas and the main laminar and interlaminar subdivisions of the nucleus cannot be decisively answered. The medium and small cells of the main laminae project primarily upon area 17, the large cells of these laminae and of the central interlaminar nucleus upon area 18, and the medial interlaminar nucleus to area 19. After survival periods of three as well as of seven days there is fibre degeneration in the medial interlaminar nucleus only when area 19 is damaged, and the findings in one brain (three-day survival) with damage of area 17 on one side and in area 18 on the other would suggest that the cortico-geniculate fibres are projecting back in a reciprocal manner. It may be noted that the observation of Guillery (1966), in Golgi-stained sections, of thicker Type 1 axons (tentatively considered as being of extraretinal origin) with larger branches in the central interlaminar plexus, would agree with this suggestion. If this interpretation of the degeneration at three days is correct, it would indicate that the cortico-geniculate projection is less than the retino-geniculate, and that it influences primarily those parts of the nucleus and cells which send their axons to that part of the cortex; the validity of this interpretation and the precise mode of termination, however, must await further electron miscroscopic studies.

The degeneration found in the ventral lateral geniculate nucleus after damage of the visual cortex confirms previous descriptions (Nauta and Bucher, 1954; Beresford, 1961; Altman, 1962) and the finding of such degeneration after three days strongly suggests that it is orthograde in nature. Although no cellular degeneration has been found in this nucleus 
after removal of the cortex, the findings in the lateral geniculate nucleus which indicate a reciprocity in thalamo-cortical connexions raises the question of a possible cortical projection from this ventral element, and it would be of interest to investigate this by means of lesions placed in it.

Projections from the visual cortex to the nucleus lateralis posterior and the posterior nucleus of the thalamus have been described by Nauta and Bucher (1954) in the rat, and by Beresford (1961) and Altman (1962) in the cat, and there are several striking features of this projection in the present material. The degeneration is very dense, is easily identifiable even after minute lesions and is present after three days. Small areas of damage confined to area 17 or to area 18 result in degeneration in the nucleus lateralis posterior and indicate that they have an independent projection. There is a distinct topographical organization present, both in the antero-posterior and medio-lateral dimension. Because no marked cell loss occurs in these nuclei after damage of the visual cortex (Sprague, 1966a; Garey and Powell, 1967) and stereotoxic lesions placed in it do not result in fibre degeneration in the lateral gyrus (Wilson and Cragg, 1967), and because the degeneration is heavy even after only three days, it is likely that this is a true corticothalamic projection. As the terminology of this region of the thalamus is confusing, it should be emphasized again that, for convenience, we have followed that of Jasper and Ajmone-Marsan (1954), and it must also be pointed out that we have not convinced ourselves that this degeneration extends sufficiently far ventrally to involve unequivocally the 'posterior group' of nuclei as defined by Rose and Woolsey (1958) and by Poggio and Mountcastle (1960); because of the difficulty of precisely delimiting the latter nuclei a different interpretation would be understandable, but at the most, only the dorsal parts of this group would be affected. On the present evidence this projection of the visual cortex upon thalamic nuclei other than the lateral geniculate may be interpreted in two distinctly different ways, each of general interest in regard to the relationship between the thalamus and the cortex. The first, and probably more reasonable, would suggest that it should be regarded as an example of a primary sensory area projecting upon an 'association' nucleus (Mettler, 1935; Peele, 1942; Jasper and Ajmone-Marsan, 1952) and would further suggest that afferent information to the relevant 'association' cortex from the sensory pathway is mediated through the 'association' thalamic nucleus as well as through intercortical connexions. It is of interest that in the olfactory pathway there is a projection from the pyriform cortex to another 'association' nucleus, the medio-dorsal (Powell, Cowan, and Raisman, $\stackrel{ }{\mathrm{O}}$ 1965). On the other hand, a second interpretation $Z$ could be that the nucleus lateralis posterior is $\stackrel{\Phi}{=}$ projecting upon the visual cortex, and the fibre degeneration seen in our material is indicative of a reciprocal pathway as there is now increasing evidence that individual thalamic nuclei project more widely upon the cortex than was formerly $\frac{\text { ? }}{5}$ considered (Guillery, Adrian, Woolsey, and Rose, 1966; Glickstein, King, Miller, and Berkley, 1967; Wilson and Cragg, 1967; Garey and Powell, 1967; $\Rightarrow$ Jones, 1967); the absence of retrograde cellular $\stackrel{9}{+}$ degeneration in the lateralis posterior following damage of the visual cortex does not exclude this $\frac{\bar{O}}{\bar{N}}$ possibility.

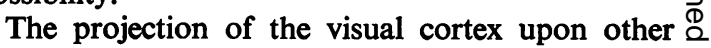
subcortical structures need not be discussed in $\%$ detail; their main significance lies in showing that $\vec{\circ}$ even quite small lesions in area 17 or 18 result in definite degeneration in sites such as the pontine $\vec{\omega}$ nuclei on which the evidence is still contradictory, or in the caudate nucleus where a projection has been demonstrated on the basis of large lesions (Webster, 1965). It may be noted that. as the cortico-pontine fibres from the visual cortex end $i$ in the same elements of the pontine nuclei as d $\vec{\Phi} \vec{\omega}$ fibres from the superior colliculus (Brodal and Jansen, 1946), there is both a direct and an indiregt cortical influence upon these cerebellar relay nucler. $\vec{\nabla}$

\section{DISCUSSION OF INTERRELATIONS}

In an important study of the projection of the neo cortex upon the brain stem of the monkey, Kuypers and Lawrence (1967) have shown that cortical areas related to particular sensory systems project to those parts of the brain stem which also receive afferent connexions from the same sensory pathways, $\stackrel{\mathbb{D}}{\varnothing}$ and they suggested that there exists a 'series of cortical $\underset{\vec{F}}{\vec{P}}$ loops each arranged in parallel with specific sub- $\frac{9}{3}$ cortical mechanisms'. As these authors pointed out, the size of their lesions precluded a detailed analysis of the organization within these systems. The present study on the organization within the connexions of the visual cortex had led us independently to a similar conclusion (Garey, 1965), and it can be regarded as complementary to the broad study of Kuypers and Lawrence (1967). These interrelationships between the cortical and subcortical parts of the visual system in the cat will be discussed on the basis of the present findings and of those of other recent investigations.

The retina projects upon the superior colliculus $N$ and the lateral geniculate nucleus in such a manner that the temporal retina is related to these structures on the ipsilateral side and the nasal retina to those $C$ 
on the contralateral side, so that both the superior colliculus and the lateral geniculate nucleus of one side are activated by stimuli in the contralateral visual field. In each of these projections there is a well-defined pattern of organization from each half of the retina, and in the lateral geniculate nucleus there appears to be a triple representation of each part of the retina, in the main laminae, and in the central and medial interlaminar nuclei (Apter, 1945; Bishop, Kozak, Levick, and Vakkur, 1962; Seneviratne and Whitteridge, 1962; Stone and Hansen, 1966; Laties and Sprague, 1966; Garey and Powell, 1968). The lateral geniculate nucleus in turn projects differentially upon the three architectonic (and functional) subdivisions of the visual cortex and also upon the cortex of the middle suprasylvian sulcus (Glickstein et al., 1967; Wilson and Cragg, 1967; Garey and Powell, 1967), and, as the constituent parts of the nucleus which are related to the three architectonic areas are essentially the same as those related to the separate representations of the retina, it is possible that there may be three more or less separate pathways from the retina to the cortex.

Four areas of cortex then receive a direct projection from the lateral geniculate nucleus, and a precise representation of the retina has been found in three of these (Hubel and Wiesel, 1965). To what extent the functional differences of the cells of these areas may be attributed to the differences in afferent connexions from the geniculate nucleus or to the substantial association cortical connexions between them must remain speculative. Area 17 sends association fibres to areas 18 and 19 and to the lateral suprasylvian cortex, while area 18 sends to areas 17, 19, and the same areas of the suprasylvian gyrus, but whether all the areas are reciprocally connected is not known, as small lesions have not been placed in either area 19 or in the suprasylvian cortex. The cortex of area 6 appears to be the only non-visual area to which areas 17 and 18 project, but it is possible that the association fibres from area 19 and the lateral suprasylvian cortex are differently distributed. Most of the interconnexions made by the association fibres are between areas whose cells have been activated by stimuli in the same visual field, but the callosal connexions serve as a link between the two retino-cortical pathways which have diverged at the chiasma. But, whereas all parts of the architectonic areas which have heen investigated appear to send, and receive, association fibres and the latter spread widely in an anteroposterior direction, the callosal fibres are fewer and terminate over a more restricted antero-posterior and medio-lateral extent, perhaps only within the area of representation of the vertical meridian and adjoining parts of the retina. In the cortex, therefore, there are considerable interconnexions between the areas receiving fibres from the lateral geniculate nucleus, but surprisingly few to, or from, other areas, and the only connexion between the representation of the ipsilateral and contralateral visual fields is restricted to those parts adjoining the vertical meridian.

If this brief analysis of the ascending and cortical connexions of the visual cortex is relevant to the sequence of function in the visual pathway, it would appear that considerable and highly ordered integration is possible between the separate cortical representations of the contralateral visual field. In contrast, it would appear that integration is possible between specific parts only of the ipsilateral and contralateral fields and that virtually none occurs-with the possible exception of connexions from the lateral suprasylvian cortexbetween the visual areas and other sensory systems. To a large extent the same would appear to be true of the projection of the visual area upon subcortical structures, but, because of the other connexions of the latter, integration with other sensory pathways is possible at this level.

Those efferent pathways from the visual cortex which have been studied here have been found to terminate in many diverse sites, which may be broadly grouped into those within the forebrain on the one hand and those in the brain-stem on the other. In each of these two groups certain of the structures which receive fibres from the visual cortex also receive a direct afferent supply from the retina while others do not; it is of interest that in both the brain-stem and in the thalamus certain of the structures which do not receive optic tract fibres nevertheless receive indirectly a dual innervation from the visual pathway. The lateral geniculate nucleus and the superior colliculus are the principal sites of termination of optic tract fibres and also probably of efferent fibres from the visual cortex. The projections of the cortex upon these two areas are similar in that they are both precisely organized so that any part of the cortex related to a particular part of the retina sends fibres only to those parts of the lateral geniculate nucleus and superior colliculus which are directly related to the same part of the retina. A further similarity is that each receives a projection from more than one of the structural and functional subdivisions of the visual cortex. They differ, however, in that the projection to the lateral geniculate nucleus constitutes a reciprocal 'feed-back' mechanism to a relay nucleus within the same sensory pathway, whereas that to the superior colliculus is to the other major site of retinal projection, the mesencephalic, and it thereby forms a major link between the two 

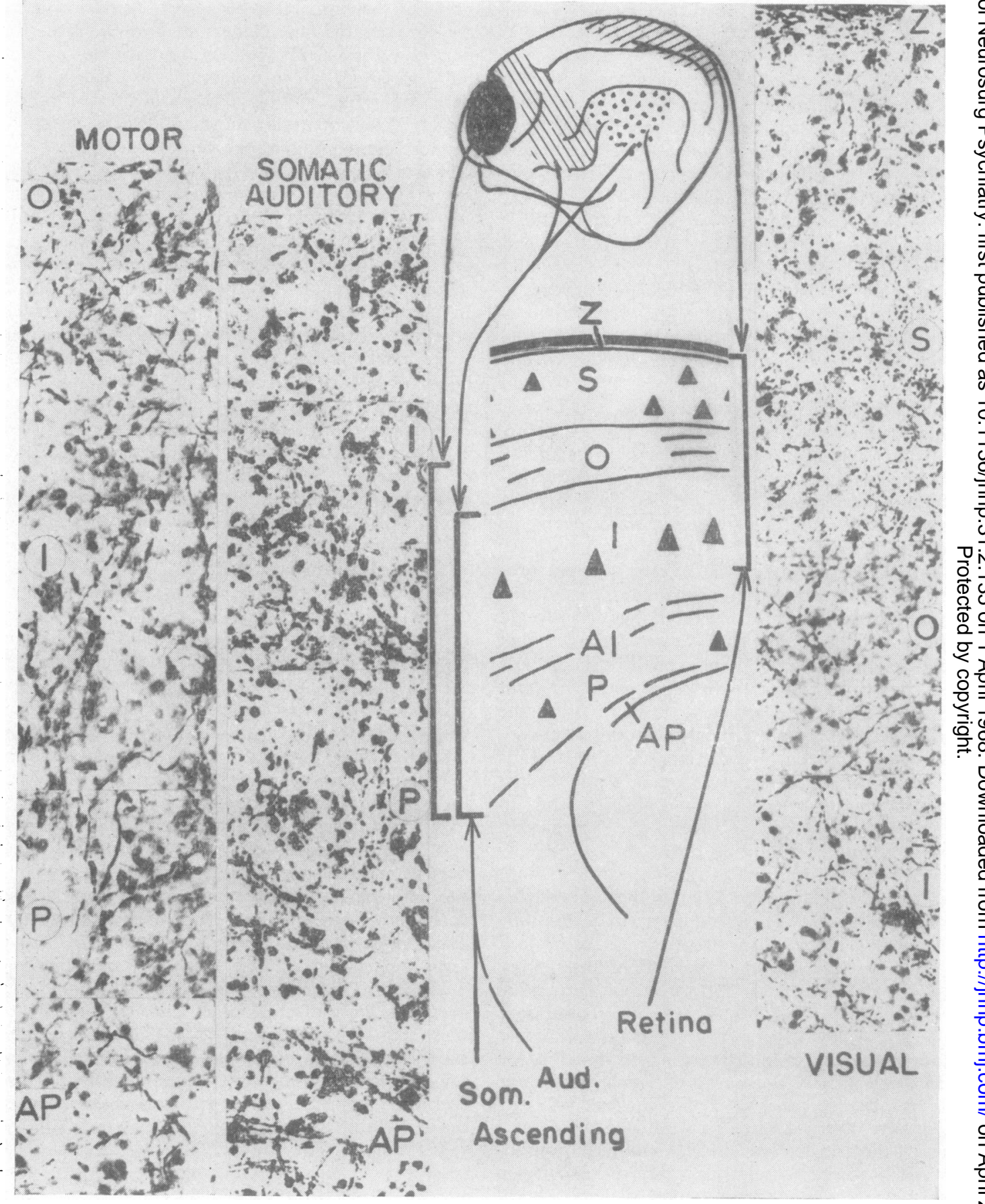

FIG. 13. To summarize the laminar distribution of the termination of the corticotectal fibres from the main 0 functional areas of the cortex, and to show the interrelationship of those from sensory areas with ascending $N$ fibres of the same pathway. The photomicrographs are montages of small areas of the relevant laminae (indicated by the vertical bars) to show degeneration after damage of the relevant cortical areas. $\times 520$. 
subdivisions of the central connexions of the retina. This difference between the projections of the visual cortex is emphasized by the fact that the efferent fibres from the three architectonic areas to the lateral geniculate nucleus pass to different elements of the nucleus, but in the case of the colliculus they all terminate in the same layers. It is probable, too, that the projection upon the colliculus is heavier than that to the geniculate.

The termination of fibres from the retina and of those from the visual cortex in essentially the same layers of the colliculus means that the same cells, or closely adjacent ones, receive distinctly different types of 'information'-that of ganglion cell activity on the one hand, and that of a more highly integrated and complex nature from cortical cells on the other. This relationship between the two sets of afferent fibres from the visual pathway is not unique, however, as the same appears to be true in the deeper layers of the colliculus in respect to the termination of fibres from the cortical and peripheral parts of the somatic and auditory sensory systems (Fig. 13). Thus, at one level of the colliculus there is clearly the possibility of integration between peripheral and cortical activity within a sensory system (Jassik-Gerschenfeld, Ascher, and Guevara, 1966) similar to that served by association connexions between the functional subdivisions of the visual cortex, and there is the further possibility of interaction between different sensory systems (Jassik-Gerschenfeld, 1966) by interconnexions between the layers. This pattern of fibres from a particular sensory area of cortex terminating upon cells which also receive fibres from more peripheral levels is also seen in the ventral lateral geniculate nucleus and the pretectum, and is in agreement with the findings of Kuypers and Lawrence (1967), who found a similar relationship between the cortical and ascending fibres of other sensory pathways for other nuclei of the brain-stem as well.

The significance of the projection of the visual cortex upon other sites, and of other possible interrelationships will not be considered in detail, but the projections to the nucleus lateralis posterior of the thalamus and to the pontine nuclei are of interest because they have features in common which may suggest certain principles of cortico-subcortical connexions which may in turn extend those proposed by Kuypers and Lawrence (1967). These two sites, one in the thalamus and the other in the brain-stem, each receive fibres from the visual cortex, and, although neither receives fibres from the retina, both have been described as receiving fibres from the superior colliculus (Brodal and Jansen, 1946; Altman and Carpenter, 1961). It is possible, therefore, that these are sites of further integration of visual information of cortical and collicular origin, and it is noteworthy that one projects to the cerebellar cortex and the other to 'association' cortex of the suprasylvian gyrus.

There is now good evidence to indicate that the superior colliculus is important for functions other than its well-known role in the control of eyemovements, and in particular the work of Myers (1961), Denny-Brown (1962), and Sprague and Meikle (1965) has shown its relationship to visual attention and perception. Recent studies on the anatomical connexions of the colliculus support these suggestions (Meikle and Sprague, 1964; Sprague and Meikle, 1965) and certain points only will be considered here. As the superior colliculus receives afferent fibres from cortical and peripheral levels of many sensory systems (Fig. 13) and as there is a precise degree of organization in one of these, there is, first, the possibility of highly ordered integration between complex cortical activity with the simpler information from the periphery within a particular sensory system. Secondly, because of the laminar overlap in the connexions and the existence of interconnexions between the laminae, further correlation between these systems is possible. If so, it is of particular relevance to the visual system that responses from proprioceptors in the eye muscles have been recorded in the deep layers (Fillenz, 1955; Cooper et al., 1955) and responses from stimulation of the retina in superficial layers. Finally, in view of the role of the superior colliculus in visually guided behaviour it is significant that the termination of the fibres to the colliculus from the motor cortex overlap the laminae to which fibres from the other sensory system project (Fig. 13). Like the neocortex, the colliculus is clearly laminated and complex in structure, but it would appear to differ in that the different sensory systems terminate at different depths in the colliculus, whereas in the cortex they terminate in separate areas. Although the structure of the superior colliculus is less complex in mammais as compared with the optic tectum of lower vertebrates, the presence of such a highly ordered projection from the visual and other sensory areas of cortex would indicate that its functional role may have changed, but be no less important. Furthermore, its widespread efferent connexions would suggest its importance; of its ascending connexions those to the nucleus lateralis posterior and medio-dorsal nucleus of the thalamus are of particular interest in suggesting not only that this may be the main pathway of visual information to other areas of cortex, but also that these areas receive integrated activity from both the thalamocortical and mesencephalic subdivisions of the retinal projection; through its descending con- 
nexions collicular activity can be passed not only to the brain-stem and spinal cord, but also to the cerebellum and possibly to the retina (Brooke, Downer, and Powell, 1965). The possible functional significance of its commisural connexion has only recently been considered (Sprague, 1966b), and whether these are similar to the callosal connexions of the visual cortex in connecting only specific parts of the representation of the retina remains to be determined. In any case parts of the colliculus containing the representation of the midline differ from other parts of the colliculus in that they alone receive afferents from callosally connected parts of cortex.

\section{SUMMARY}

The projection of the visual and other areas of the neocortex upon the superior colliculus, together with the cortical and other subcortical connexions of the visual cortex, have been studied in the cat using the Nauta method. Areas 17 and 18 of the visual cortex have an independent and wellorganized projection to the superficial layers of the colliculus, so that a part of the retina is related to these layers of a particular part of the colliculus directly and also indirectly through the thalamus and cortex. Other areas of the cortex also send efferent fibres to the colliculus; those from the auditory and somatic sensory areas end in the same layers as fibres from their respective ascending pathways; the termination of the fibres from the motor cortex overlaps the termination of those from the visual and other sensory systems.

The callosal fibres of the visual cortex terminate in the extreme lateral part of area 17, the medial half of area 18, the lateral part of area 19, and in the cortex of the middle suprasylvian gyrus. This pattern of termination of the callosal fibres agrees with the suggestion that they connect parts of the cortex related to the representation of the vertical meridian. Area 17 sends association fibres to areas 18 and 19, the lateral suprasylvian cortex, and area 6 , while association fibres from area 18 pass to areas 17 and 19, the same parts of the suprasylvian cortex, and area 6.

In addition to its projection upon the superior colliculus, the visual cortex sends subcortical projection fibres to the dorsal and ventral nuclei of the lateral geniculate body, the nucleus lateralis posterior and posterior nucleus of the thalamus, the pretectum and accessory nuclei of the optic tract, the caudate nucleus, and the pontine nuclei.

This work was supported by grants from the Medical Research Council and the United States Public Health Service.

\section{REFERENCES}

Altman, J. (1962). Some fiber projections to the superior colliculus in $\mathbb{D}$ the cat. J. comp. Neurol., 119, 77-95.

- and Carpenter, M. B. (1961). Fiber projections of the superior colliculus in the cat. Ibid., 116, 157-177.

Apter, J. T. (1945). Projection of the retina on superior colliculus of $\frac{\bar{Q}}{a}$ cats. J. Neurophysiol., 8, 123-134.

Beresford, W. A. (1961). Fibre degeneration following lesions of the visual cortex of the cat. In Neurophysiologie und Psychophysik des visuellen Systems, edited by R. Jung, and H. Kornhuber, $\frac{\rho}{\supset}$ pp. 247-255. Springer, Berlin.

-, (1963). The anatomical connections of the visual cortex. D. Phil. Thesis, University of Oxford.

Bilge, M., Bingle, A., Seneviratne, K. N., and Whitteridge, D. (1967). A map of the visual cortex in the cat. J. Physiol.(Lond.), 191,

116P-118P.
- Seneviratne, K. N., and Whitteridge, D. (1963). The primary visual receptive area of the cerebral cortex in the cat. J. Physiol. $\bar{O}$ (Lond.), 169, 36P

Bishop, P. O., Kozak, W., Levick, W. R., and Vakkur, G. J. (1962). $\frac{\overline{\mathcal{T}}}{\bar{T}}$ The determination of the projection of the visual field on to $\mathbb{D}$ the lateral geniculate nucleus in the cat. J. Physiol. (Lond.), 163, 503-539.

Brodal, A., and Jansen, J. (1946). The ponto-cerebellar projection in the rabbit and cat. J. comp. Neurol., 84, 31-118.

Brooke, R. N. L., Downer, J. de C., and Powell, T. P. S. (1965). Centrifugal fibres to the retina in the monkey and cat. Nature $\vec{\omega}$ (Lond.), 207, 1365-1367.

Choudhury, B. P., Whitteridge, D., and Wilson, M. B. (1965). The function of the callosal connections of the visual cortex.
Quart. J. exp. Physiol., 50, 214-219.

Cooper, S., Daniel, P. M., and Whitteridge, D. (1955). Muscle spindles $\omega$ and other sensory endings in the extrinsic eye muscles; the

connexions with the brain-stem. Brain, 78, 564-583.
Cragg, B. G. (1962). Centrifugal fibers to the retina and olfactory bul| $\overrightarrow{\mid c}$ and composition of the supraoptic commissures in the rabbir Exp. Neurol., 5, 406-427.

Denny-Brown, D. (1962). The midbrain and motor integration. Prog roy. Soc. Med., 55, 527-538.

Ebner, F. F., and Myers, R. E. (1965). Distribution of corpus callosum and anterior commisure in cat and raccoon. J. comp. Neurage
$124,353-365$.

Fillenz, M. (1955). Responses in the brainstem of the cat to stretct of extrinsic ocular muscles. J. Physiol. (Lond.), 128, 182-199.

Garey, L. J. (1965). Interrelationships of the visual cortex and superior colliculus in the cat. Nature (Lond.), 207, 1410-1411.

-, and Powell, T. P. S. (1967). The projection of the lateral geniculate nucleus upon the cortex in the cat. Proc. roy. Soc. B., 169, 107-126.

- (1968). The projection of the retina in the cat. J. Anat. (Lond.), 102, 189-222.

Glees, P. (1946). Terminal degeneration within the central nervous system as studied by a new silver method. J. Neuropath. exp. Neurol., 5, 54-59.

Glickstein, M., King, R. A., Miller, J., and Berkley, M. (1967). Cortical projections from the dorsal lateral geniculate nucleus of cats. J. comp. Neurol., 130, 55-75.

Guillery, R. W. (1959). Afferent fibres to the dorso-medial thalamic nucleus in the cat. J. Anat. (Lond.), 93, 403-419.

- (1966). A study of Golgi preparations from the dorsal lateral geniculate nucleus of the adult cat. J. comp. Neurol., 128, 21-49.

__ Adrian, H. O., Woolsey, C. N., and Rose, J. E. (1966). Activation of somatosensory areas 1 and 11 of cat's cerebral cortex by focal stimulation of the ventrobasal complex. In The Thalamus, edited by D. P. Purpura, and M. D. Yahr, pp. 197-207. Columbia University Press, New York and London.

Hassler, R. (1966). Comparative anatomy of the central visual systems in day- and night-active primates. In Evolution of the Forebrain, edited by R. Hassler, and H. Stephan. Thieme, Stuttgart.

Hubel, D. H., and Wiesel, T. N. (1965). Receptive fields and functional $D$ architecture in two nonstriate visual areas (18 and 19) of the cat. J. Neurophysiol., 28, 229-289.

Jasper, H. H., and Ajmone-Marsan, C. (1952). Thalamocortical $N$ integrating mechanisms. Res. Publ. Ass. nerv. ment. Dis., 30, 493-512.

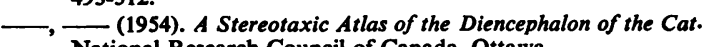
National Research Council of Canada, Ottawa. 
Jassik-Gerschenfeld, D. (1966). Activity of somatic origin evoked in the superior colliculus of the cat. Exp. Neurol., 16, 104-118.

cortical system on visual responses of the superior colliculus. Arch. ital. Biol., 104, 30-49.

Jones, E. G. (1967). The pattern of cortical and thalamic connexions of the somatic sensory cortex. Nature (Lond.), 216, 704-705.

Kuypers, H. G. J. M., and Lawrence, D. G. (1967). Cortical projections to the red nucleus and the brain stem in the rhesus monkey. Brain Res., 4, 151-188.

_-, Szwarcbart, M. K., Mishkin M., and Rosvold, H. E. (1965). Occipitotemporal corticocortical connexions in the rhesus monkey. Exp, Neurol., 11, 245-262.

Laties, A. M., and Sprague, J. M. (1966). The projection of optic fibers to the visual centers in the cat. J. comp. Neurol., 127, 35-70.

Lund, R. D. (1964). Terminal distribution in the superior colliculus of fibres originating in the visual cortex. Nature (Lond.), 204, 1283-1285.

- (1966). The occipitotectal pathway of the rat. J. Anat. (Lond.), $100,51-62$.

McGill, J. I., Powell, T. P. S., and Cowan, W. M. (1966). The retinal representation upon the optic tectum and isthmo-optic nucleus in the pigeon. Ibid., 100, 5-33.

Mehler, W. R. (1962). The anatomy of the so-called 'Pain Tract' in man: An analysis of the course and distribution of the ascending fibres of the fasciculus anterolateralis. In Basic Research in Paraplegia, edited by J. D. French, and R. W. Porter. Thomas, Springfield, Ill.

Meikle, T. H., and Sprague, J. M. (1964). The neural organization of the visual pathways in the cat. Int. Rev. Neurobiol., 6, 149-189.

Mettler, F. A. (1935). Corticofugal fiber connections of the cortex of Macaca mulatta. The parietal region. J. comp. Neurol., 62, 263-291.

Moore, R. Y., and Goldberg, J. M. (1963). Ascending projections of the inferior colliculus in the cat. J. comp. Neurol., 121, 109-135.

Exp. Neurol., 14, 429-438.

Myers R. E. (1961). Perceptual effects of tectal lesions. Fed. Proc., 20, 336.

(1962). Commissural connections between occipital lobes of the monkey. J. comp. Neurol., 118, 1-16.

Nauta, W. J. H., and Bucher, V. M. (1954). Efferent connections of the striate cortex in the albino rat. J. comp. Neurol., 100, 257-286.

-, and Gygax, P. A. (1954). Silver impregnation of degenerating axons in the central nervous system. A modified technic. Stain Technol., 29, 91-93.

-, and Kuypers, H. G. J. M. (1958). Some ascending pathways in the brain stem reticular formation. In Reticular Formation of the Brain, edited by H. H. Jasper, L. D. Proctor, R. S. Knighton W. C. Noshay, and R. T. Costello. (Henry Ford Hospital International Symposium, 1957), pp. 3-30. Little, Brown, Boston.
Otsuka, R., and Hassler, R. (1962). Uber Aufbau und Gliederung der corticalen Sehspare bei der Katze. Arch. Psychiat. Nervenbr., 203, 212-234

Peele, T. L. (1942). Cytoarchitecture of individual parietal areas in the monkey (Macaca mulatta) and the distribution of the efferent fibers. J. comp. Neurol, 77, 693-737.

Poggio, G. F., and Mountcastle, V. B. (1960). A study of the functional contribution of the lemniscal and spinothalamic systems to somatic sensibility. Bull. Johns Hopk. Hosp., 106, 266-316.

Powell, T. P. S., and Cowan, W. M. (1964). A note on retrograde fibre degeneration. J. Anat. (Lond.), 98, 579-585.

- - and Raisman, G. (1965). The central olfactory connexions. Ibid., 99, 791-813.

Rose, J. E., and Woolsey, C. N. (1958). Cortical connections and functional organisation of the thalamic auditory system of the cat. In Biological and Biochemical Bases of Behavior, edited by H. R. Harlow, and C. N. Woolsey. pp. 127-150, Univ. Wisconsin Press, Madison.

Seneviratne, K. N., and Whitteridge, D. (1962). Visual evoked responses in the lateral geniculate nucleus. Electroenceph. clin. Neurophysiol, 14, 785.

Sprague, J. M. (1963). Corticofugal projections to the superior colliculus in the cat. Anat. Rec., 145, 288.

- (1966a). Visual, acoustic, and somesthetic deficits in the cat after cortical and midbrain lesions. In The Thalamus, edited by D. P. Purpura and M. D. Yahr. pp. 391-417. Columbia University Press, New York and London.

- (1966b). Interaction of cortex and superior colliculus in mediation of visually guided behavior in the cat. Science, 153, 1544-1547.

-, and Meikle, T. H. (1965). The role of the superior colliculus in visually guided behavior. Exp. Neurol., 11, 115-146.

Stone, J. (1966). The naso-temporal division of the cat's retina. J. comp. Neurol., 126, 585-600.

_ and Hansen, S. M. (1966). The projection of the cat's retina on the lateral geniculate nucleus. Ibid., 126, 601-624.

Szentágothai, J., Hámori, J., and Tömböl, T. (1966). Degeneration and electron microscope analysis of the synaptic glomeruli in the lateral geniculate body. Exp. Brain Res., 2, 283-301.

Talbot, S. A., and Marshall, W. H. (1941). Physiological studies on neural mechanisms of visual localization and discrimination. Amer. J. Ophthal., 24, 1255-1264.

Webster, K. E. (1965). The cortico-striatal projection in the cat. J. Anat. (Lond.), 99, 329-337.

Widén, L., and Ajmone-Marsan, C. (1960). Effects of corticipetal and corticifugal impulses upon single elements of the dorsolateral geniculate nucleus. Exp. Neurol., 2, 468-502.

Wilson, M. E., and Cragg, B. G. (1967). Projections from the lateral geniculate nucleus in the cat and monkey. J. Anat. (Lond.), 101, 677-692.

Woollard, H. H., and Harpman, J. A. (1940). The connexions of the inferior colliculus and of the dorsal nucleus of the lateral lemniscus. Ibid., 74, 441-458. 